\title{
Surface reaction and transport in mixed conductors with electrochemically-active surfaces: a 2-D numerical study of ceria
}

\author{
Francesco Ciucci, $\dagger_{\dagger}^{a}$ William C. Chueh, $\dagger^{b}$ David G. Goodwin ${ }^{a}$ and \\ Sossina M. Haile ${ }^{* b}$
}

Received 16th July 2010, Accepted 7th September 2010

DOI: $10.1039 / \mathbf{c 0 c p 0 1 2 1 9 j}$

\begin{abstract}
A two-dimensional, small-bias model has been developed for describing transport through a mixed ionic and electronic conductor (MIEC) with electrochemically-active surfaces, a system of particular relevance to solid oxide fuel cells. Utilizing the h-adaptive finite-element method, we solve the electrochemical potential and flux for both ionic and electronic species in the MIEC, taking the transport properties of $\mathrm{Sm}_{0.15} \mathrm{Ce}_{0.85} \mathrm{O}_{1.925-\delta}$ (SDC15). In addition to the ionic flux that flows between the two sides of the cell, there are two types of electronic fluxes: (1) cross-plane current that flows in the same general direction as the ionic current, and (2) in-plane current that flows between the catalytically-active MIEC surface and the metal current collectors. From an evaluation of these fluxes, the macroscopic interfacial resistance is decomposed into an electrochemical reaction resistance and an electron diffusion-drift resistance, the latter associated with the in-plane electronic current. Analysis of the experimental data for the interfacial resistance for hydrogen electro-oxidation on SDC15 having either Pt or Au current collectors (W. Lai and S. M. Haile, J. Am. Ceram. Soc., 2005, 88, 2979-2997; W. C. Chueh, W. Lai and S. M. Haile, Solid State Ionics, 2008, 179, 1036-1041) indicates that surface reaction rather than electron migration is the overall rate-limiting step, and suggests furthermore that the surface reaction rate, which has not been directly measured in the literature, scales with $p_{\mathrm{O}_{2}}^{-1 / 4}$. The penetration depth for the in-plane electronic current is estimated at $0.6 \mu \mathrm{m}$ for the experimental conditions of interest to SDC15, and is found to attain a value as high as $4 \mu \mathrm{m}$ within the broader range of computational conditions.
\end{abstract}

\section{Introduction}

Solid-state mixed ionic and electronic conductors (MIECs) have received significant attention as candidate materials for solid oxide fuel cell (SOFC) components, permeation membranes, oxygen storage capacitors, electrochemical sensors, active catalyst support, etc. ${ }^{1-4}$ As fuel cell electrodes, MIECs have the potential to broaden the electrochemical reaction zone beyond the triple-phase boundary at which electrode, electrolyte and gas phase are in simultaneous contact. That is, in a conventional fuel cell, electrochemical reactions are limited to triple-phase boundaries because only to and from such locations can ions, electrons and gas molecules each be transported. Recognition of this inherent advantage of MIECs has driven efforts to develop oxides with both high ionic and high electronic conductivity as solid oxide fuel cell anodes and/or cathodes. ${ }^{1,4-7}$ It is further recognized that, in addition to high bulk diffusion rates, high surface activity is also essential for high performance from an oxide electrode. ${ }^{1,4-7}$ What remains unclear are the relative

${ }^{a}$ California Institute of Technology, Division of Engineering \& Applied Sciences, Pasadena, CA 91125, USA.

E-mail: smhaile@caltech.edu

${ }^{b}$ California Institute of Technology, Materials Science, Pasadena, CA 91125, USA

$\dagger$ Equally contributing authors.

$\ddagger$ Present address: Universität Heidelberg, Interdisciplinary Center for Scientific Computing, D-69120 Heidelberg, Germany contributions of reaction and diffusion kinetics to the overall interfacial impedance associated with MIECs, and the role that microstructure may play in balancing these factors. This work presents a numerical study of an idealized electrode geometry for addressing these questions.

There have been extensive modeling efforts focusing on surface reaction-transport interactions in MIECs. ${ }^{8-28}$ Many employ porous medium theory and effective transport parameters to reduce the systems from three to one dimension. ${ }^{8,15,20,24,25,28}$ While such one-dimensional models are inherently unable to treat local potential and flux distributions at small length scales, they are directly applicable to certain geometries or materials systems (e.g. thin-film and thin-column electrodes with homogenous properties) and hence have been useful under appropriate conditions for decoupling surface reaction and bulk diffusion kinetics. ${ }^{9,21,22,29}$ Beyond these effective medium models, a handful of twodimensional current-voltage and impedance models have been developed to quantify the behavior of a wider range of electrode structures, with the majority of them focused on SOFC cathode materials in which the electronic conductivity greatly exceeds that of the ions. ${ }^{14,17-19,26,27}$ In addition, both the one- and two-dimensional MIEC electrode models involve a pure ionic conductor support, in which the electron current is confined within the electrode. Growing interest in the SOFC community to use MIECs with high ionic conductivity and acceptable levels of electronic conductivity (e.g. doped $\mathrm{CeO}_{2}$, 
or ceria) both as electrolytes and as components in metalMIEC composite electrodes, motivate the development of a generalized model to investigate transport of ionic and electronic carriers in the bulk and near interfaces.

In this work, we consider a geometry in which a MIEC is placed between porous (patterned) metal current collectors and the entire cell is held under a uniform gas atmosphere. Electrochemical reactions at the MIEC surfaces facilitate current flow, which is driven by an applied electric potential. The MIEC serves as the both the redox-active catalyst for the electrochemical reaction, and the electrolyte through which ions are transported. It is to be emphasized that mixed conductivity is not, in itself, a sufficient condition to ensure high surface catalytic activity; the aim here is to explore the relative roles of reaction rate and electron transport rate given an active MIEC. Furthermore, while the particular geometry studied is physically symmetric, the model and results can be extended to an asymmetrical gas environment (i.e. a half-cell), as is encountered in an operational fuel cell. We solve for the electrochemical potential and flux for both ions and electrons, which are coupled via the electric potential. Treatment of the problem inherently requires multidimensional modeling tools in order to adequately describe the complexity of electrochemical potential and flux distributions near the MIEC/gas and the MIEC|metal interfaces. We focus our analysis on decoupling surface reaction and bulk electron conduction near the surface. For electrochemical reactions to occur on the MIEC/gas interface, electrons need to migrate from the reaction site to the external circuit (and vice versa) via the metal current collector. Such a step, termed in-plane electron diffusion-drift, could play a significant role in the interfacial behavior of mixed conductors, particularly for those exhibiting a low to moderate bulk electronic conductivity and high surface catalytic activity. ${ }^{27}$ In the present system, the in-plane diffusion-drift resistance is impossible to estimate using one-dimensional approximations as the electron penetration depth is not known.

The material system selected for this investigation is metal $\left|\mathrm{Sm}_{0.15} \mathrm{Ce}_{0.85} \mathrm{O}_{1.925-\delta}(\mathrm{SDC} 15)\right|$ metal, and the computational results are utilized to interpret the electrochemical measurements by Lai and Haile ${ }^{23}$ and Chueh et al. ${ }^{30}$ High oxygen ion conductivity of acceptor-doped ceria at intermediate temperatures $\left(\sigma_{\text {ion }} \approx 10^{-2} \mathrm{~S} \mathrm{~cm}^{-1} \text { at } 550{ }^{\circ} \mathrm{C} \text { in air }\right)^{23}$ renders this material particularly attractive as a fuel cell electrolyte. Moreover, under mildly reducing condition, doped ceria exhibits a moderate electronic conductivity $\left(\sigma_{\text {eon }} \approx\right.$ $10^{-2} \mathrm{~S} \mathrm{~cm}^{-1}$ at $550{ }^{\circ} \mathrm{C}, p_{\mathrm{O}_{2}}=10^{-25}$ atm), making it also useful for fuel cell anode applications. The high surface activity of doped ceria is suggested by the observation that the electrochemical reaction rate for hydrogen electrooxidation at the metal/ceria interface is relatively independent of the choice of metal. ${ }^{30}$ Recent studies have further shown that ceria-based anodes are largely resistant to carbon coking in the presence of hydrocarbon fuels..$^{3,31-32}$ Beyond deepening our understanding of reaction-transport coupling in MIECs, insight into the rate-limiting step in ceria-based electrodes could lead to improved designs of anode geometries and reduced interfacial resistance. Only by utilization of multidimensional models as developed here can one rigorously separate the impact of various components of the polarization losses.

\section{Physical system}

The physical model, depicted in Fig. 1(a), consists of a MIEC conductor (doped ceria) with patterned metal current collectors ( $\mathrm{Pt}$ or $\mathrm{Au}$ ) on both sides, placed in a uniform gas environment $\left(\mathrm{H}_{2}-\mathrm{H}_{2} \mathrm{O}-\mathrm{Ar}\right.$ mixture). The patterned metal current collectors permit the system to be described, Fig. 1(b), by repeating domains using mirror symmetry lines $\left(\Gamma_{2}\right.$ and $\left.\Gamma_{3}\right)$. The thickness of the cell is fixed at $2 l=1 \mathrm{~mm}$. Mirror symmetry along $\Gamma_{1}$ implies the computational domain can be further limited to one-half this total thickness. The current collector dimensions, the width of the MIEC|metal interface ( $\Gamma_{4}$ boundary), $2 W_{1}$, and the width of the MIEC $\mid$ gas interface $\left(\Gamma_{5}\right.$ boundary), $2 W_{2}$, are, unless stated otherwise, fixed at values of 3 and $5 \mu \mathrm{m}$, respectively. These dimensions are selected to approximate a typical electrochemical experiment in which a MIEC is placed between porous metal current collector with $5 \mu \mathrm{m}$ pores and $3 \mu \mathrm{m}$ MIEC|metal contact regions.

Upon application of a potential bias across the oxide, electrical current flows, carried primarily by ions as the ionic transference number in ceria under typical temperature and oxygen partial pressures is greater than 0.5 . Transport by ions, in turn, implies the occurrence of electrochemical reactions at the MIEC|gas interface, written for the forward case as

$$
\mathrm{H}_{2}(\mathrm{~g})+\mathrm{O}_{\mathrm{O}}^{\times} \rightarrow \mathrm{H}_{2} \mathrm{O}(\mathrm{g})+\mathrm{V}_{\mathrm{O}}^{\bullet \bullet}+2 \mathrm{e}^{\prime}
$$


Fig. 1 (a) Schematic of the electrochemical cell modeled: a symmetric cell with patterned metal current collectors on both sides of a MIEC placed in a uniform gas atmosphere. (b) The modeling domain with the boundaries shown: $\Gamma_{1}, \Gamma_{2}$, and $\Gamma_{3}$ are symmetry lines, $\Gamma_{4}$ is the MIEC $\mid$ metal interface, and $\Gamma_{5}$ is the MIEC|gas interface. $2 W_{1}$ is the width of the metal current collector stripe, $2 W_{2}$ is the distance between each current collector stripe, and $2 l$ is the thickness of the sample. Not drawn to scale. 
where Kröger-Vink notation has been employed. The mixed conducting nature of the oxide ensures that electrochemical reactions occurring at the MIEC|gas double-phase boundary (2PB), even substantially removed from the MIEC|metal|gas triple-phase boundary (3PB), will contribute to the overall reaction flux. The inherently higher reaction area associated with the double-phase boundaries implies that, for the relatively coarse structures examined here, the contribution of the 3PBs (with effective reaction widths of no more than several nanometres) to the electrochemical reaction can, to a first approximation, be neglected, despite the likelihood of enhanced activity at these boundaries. The analysis is further simplified by taking the MIEC|metal and MIEC|gas interfaces to be reversible to electrons, by ignoring interfacial spacecharge effects and charge double-layers, and by treating the electrochemical reaction, eqn (1), in terms of a single, global step, without consideration of detailed mechanisms. The use of a global reaction constant is an operational definition and does not impact the analysis of interest here. The inevitable modification of the electric potential at interfaces can be considered, to a first approximation, to be reflected in the value of the global reaction rate and need not be directly modeled. Finally, electrochemical impedance spectroscopy results suggest that electron transfer across the doped ceria $\mid$ metal interfaces is extremely rapid relative to the formation or breaking of chemical bonds, justifying an assumption of electron reversibility for the present analysis. ${ }^{23,30}$ In combination, this set of simplifications allows examination of the two steps of interest in the electrochemical reaction pathway: the surface reaction, and the electron diffusion-drift from the active site to the metal current collector.

\section{Model}

The two-dimensional (non-steady-state) model developed to address the geometry of Fig. 1 has been presented in brief elsewhere. ${ }^{12}$ The model, described in full here, adopts the following sequence of steps. First, the carrier concentrations are solved analytically under equilibrium conditions (section 3.1). Next, transport induced by a small-bias perturbation is described using Nernst-Planck and Poisson equations (section 3.2). Subsequently, appropriate boundary conditions describing the interfaces are applied (section 3.3). Finally, carrier concentrations, flux, and electric potential are solved numerically using an adaptive finite-element method (section 3.4).

\subsection{Bulk defect chemistry: equilibrium conditions}

For acceptor (e.g. trivalent) doped ceria examined under moderate to reducing conditions, the relevant charged defects are dopants, oxygen vacancies, and localized electrons, ${ }^{33-37}$ denoted by the subscripts "dop", "ion", and "eon", respectively. At the moderate temperatures pertinent to this work, the dopant species are immobile, leaving oxygen vacancies and localized electrons as the charge carriers. The electrochemical potential $\tilde{\mu}_{i}$ of each species $i$ is given as:

$$
\tilde{\mu}_{i}=\mu_{i}+e z_{i} \phi
$$

where $\mu_{i}$ and $z_{i}$ are the chemical potential and charge of species $i$, respectively, $e$ is the electron charge, and $\phi$ is the electric potential. Under the dilute solution limit, the chemical potential can be written as

$$
\mu_{i}=\mu_{i}^{0}+k_{\mathrm{B}} T \ln \frac{c_{i}}{c_{i}^{0}}
$$

where $\mu_{i}^{0}$ is the chemical potential of species $i$ in its standard concentration $c_{i}^{0}, c_{i}$ is the concentration, $k_{\mathrm{B}}$ is Boltzmann's constant, and $T$ is temperature. In the absence of an external electric field, the equilibrium defect concentration is governed by the chemical reaction:

$$
2 \mathrm{Ce}_{\mathrm{Ce}}^{\times}+\mathrm{O}_{\mathrm{O}}^{\times} \rightleftharpoons \frac{1}{2} \mathrm{O}_{2}(\mathrm{~g})+\mathrm{V}_{\mathrm{O}}^{\bullet \bullet}+2 \mathrm{Ce}_{\mathrm{Ce}}^{\prime}
$$

where, again, Kröger-Vink notation is used, $\mathrm{Ce}_{\mathrm{Ce}}^{\prime}$ represents the cerium cation in the $3+$ oxidation state occupying the nominally cerium $4+$ site, or equivalently, a localized electron (polaron). At equilibrium, the change in chemical potential across the reaction is zero. Accordingly, the following equilibrium expression can be written for reaction (4):

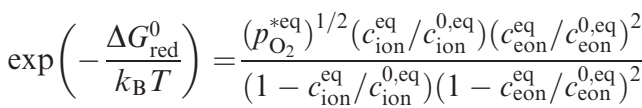

$$
\begin{aligned}
& \approx\left(p_{\mathrm{O}_{2}}^{* \mathrm{eq}}\right)^{1 / 2} c_{\text {ion }}^{* \mathrm{eq}}\left(c_{\mathrm{eon}}^{* \mathrm{eq}}\right)^{2}
\end{aligned}
$$

where $\Delta G_{\text {red }}^{0}$ is the standard Gibbs free energy of reduction, $p_{\mathrm{O}_{2}}^{*}$ is the activity of the gaseous oxygen (taken to be the ratio of the partial pressure of oxygen to the standard state of $1 \mathrm{~atm}), c_{i}^{*}$ is the fraction of crystallographic sites occupied by defects, $c_{i}^{0}$ is the standard state for solid state defects, taken to correspond to all available crystallographic sites, and the superscript 'eq' indicates equilibrium values. Under the simulation conditions, the defect fractions are much less than unity, allowing the denominator to be approximated as 1 . In addition, the gas phase reaction and equilibrium condition can be written as:

$$
\begin{aligned}
\mathrm{H}_{2}(\mathrm{~g})+\frac{1}{2} \mathrm{O}_{2}(\mathrm{~g}) & \rightleftharpoons \mathrm{H}_{2} \mathrm{O}(\mathrm{g}) \\
\exp \left(-\frac{\Delta G_{g}^{0}}{k_{\mathrm{B}} T}\right) & =\frac{p_{\mathrm{H}_{2} \mathrm{O}}^{* \mathrm{eq}}}{p_{\mathrm{H}_{2}}^{* \mathrm{eq}}\left(p_{\mathrm{O}_{2}}^{* \mathrm{eq}}\right)^{1 / 2}}
\end{aligned}
$$

In the absence of equilibrium space-charge effects, local charge neutrality is obeyed everywhere in the MIEC:

$$
\sum_{i} z_{i} c_{i}^{\mathrm{eq}}=-c_{\mathrm{eon}}^{\mathrm{eq}}+2 c_{\mathrm{ion}}^{\mathrm{eq}}-c_{\mathrm{dop}}^{\mathrm{eq}}=0
$$

This set of expressions (eqn (5), (7) and (8)), in combination with the reported values of the thermodynamic parameters for reactions (4) and (6) $\left(\Delta G_{\text {red }}^{0}(T)\right.$ and $\Delta G_{g}^{0}(T)$, respectively) enables computation of the equilibrium concentration of the charge carrier species under a given temperature and gas composition (within the limits of dilute solution behavior).

\subsection{Bulk transport}

Bulk transport occurs in response to the application of a small bias away from equilibrium. Experimentally, this is achieved by subjecting the electrochemical cell to a small voltage relative to the unbiased system. Under the assumption that 
there are no internal sources or sinks of species in the material under study, species conservation during transport implies that

$$
e z_{i} \frac{\partial c_{i}}{\partial t}+\nabla \cdot \mathbf{j}_{i}^{\text {chg }}=0
$$

where, $\mathbf{j}_{i}^{\text {chg }}$ is the charge flux of species $i$. The charge flux responds to an electrochemical potential gradient, obeying the diffusion-drift equation:

$$
\mathbf{j}_{i}^{\mathrm{chg}}=-\frac{\left(e z_{i}\right)^{2} D_{i} c_{i}}{k_{\mathrm{B}} T} \nabla \tilde{\mu}_{i}^{*}
$$

where $D_{i}$ is the diffusion coefficient and $\tilde{\mu}_{i}^{*}=\tilde{\mu}_{i} / e z_{i}$ is the reduced electrochemical potential. In the steady state case $\left(\frac{\partial c_{i}}{\partial t}=0\right)$, combining (9) and (10), and assuming that the diffusion coefficients and temperature are uniform, yields

$$
\nabla \cdot\left(c_{i} \nabla \tilde{\mu}_{i}^{*}\right)=0
$$

Finally, the carrier concentrations are coupled to the electric potential via the Poisson equation:

$$
\nabla^{2} \phi=-\frac{e}{\varepsilon} \sum_{i} z_{i} c_{i}
$$

where $\varepsilon$ is the permittivity of the material.

Upon perturbation of the system with a small voltage, the properties $\left\{q_{k}\right\}=\left\{\tilde{\mu}_{i}^{*}, c_{i}, \phi\right\}$ each adopt a value described as

$$
q_{k}=q_{k}^{\mathrm{eq}}+q_{k}^{(1)}
$$

where $q^{\text {eq }}$ is the equilibrium value and $q^{(1)}$ is the small perturbation in $q$ (such that $\left|q_{k}^{(1)}\right| \ll\left|q_{k}^{\mathrm{eq}}\right|$ ). From the assumption that the equilibrium concentration and electric potential are uniform within the system it follows that

$$
\nabla q_{k}^{\mathrm{eq}}=\nabla^{2} q_{k}^{\mathrm{eq}}=0
$$

Substituting the above definitions and properties into eqn (11), and ignoring second and higher order terms, yields the following equation

$$
\nabla^{2} \tilde{\mu}_{i}^{*(1)}=0
$$

Expanding the chemical and electric potential (eqn (2) and (3)) about their equilibrium values and ignoring higher order terms gives the following approximation for the reduced electrochemical potential under perturbation:

$$
\tilde{\mu}_{i}^{*(1)}=\frac{k_{\mathrm{B}} T}{e z_{i}} \frac{c_{i}^{(1)}}{c_{i}^{\mathrm{eq}}}+\phi^{(1)}
$$

Substituting eqn (16) into (15), in turn, yields

$$
\frac{1}{z_{i}} \nabla^{2} c_{i}^{*(1)}+\nabla^{2} \phi^{*(1)}=0
$$

where the dimensionless carrier concentration and electric potential under perturbation are defined as

$$
\begin{aligned}
c_{i}^{*(1)} & =\frac{c_{i}^{(1)}}{c_{i}^{\text {eq }}} \\
\phi^{*(1)} & =\phi^{(1)}\left(\frac{k_{\mathrm{B}} T}{e}\right)^{-1}
\end{aligned}
$$

The perturbed reduced electrochemical potential can also be written in terms of the dimensionless quantities defined above:

$$
\tilde{\mu}_{i}^{*(1)}=\frac{k_{\mathrm{B}} T}{e}\left[\frac{c_{i}^{*(1)}}{z_{i}}+\phi^{*(1)}\right]
$$

Eqn (17), together with the Poisson equation, represents a system of coupled nonlinear differential equations. The evaluation is simplified by making use of the electroneutrality condition (eqn (8)) which links the equilibrium ionic and electronic carrier concentrations. A small perturbation in the concentration of one carrier must be accompanied by a change in the concentration for the other carrier. For the specific case of doped ceria system, this relationship is:

$$
2 c_{\mathrm{ion}}^{(1)} \approx c_{\mathrm{eon}}^{(1)}
$$

Writing eqn (17) for both ionic and electronic charge carriers and applying the above approximation gives:

$$
\begin{aligned}
& \nabla^{2} c_{\mathrm{eon}}^{*(1)}=0 \\
& \nabla^{2} \phi^{*(1)}=0
\end{aligned}
$$

Under a small perturbation, carrier concentration and electric potential obey the partial differential equations eqn (22) and (23). With the application of appropriate boundary conditions, the carrier concentration profile and electric potential distribution can be computed. In turn, these results give the electrochemical potential distribution and the charge flux. In principle, a non-uniform carrier concentration profile implies spatially varying conductivities. However, because the perturbation is small, this effect is sufficiently small that it can be ignored. Specifically, for a typical voltage perturbation of $50 \mathrm{mV}$ across the entire cell, the impact on electron concentration is less than a factor of two, whereas the vacancy concentration remains fixed by the dopant concentration. Furthermore, the variation in the ionic and electronic electrochemical potentials in the thin surface region impacted by electron diffusion-drift is typically less than $1 \%$ under the conditions examined.

\subsection{Boundary conditions}

As shown in Fig. 1(b), the boundary conditions along a total of five boundaries must be specified. As already noted, although the behavior of charge carriers near boundaries is complex in the real system as a result of the formation of charge double-layers, for the purposes of evaluating the relative roles of electronic transport and electrochemical reaction rates, a detailed description of these phenomena is not required. Accordingly, such effects are ignored here.

By symmetry arguments, electric potential and carrier concentration do not vary along the mirror symmetry line $\Gamma_{1}$ and current flows only along the direction perpendicular to the boundary. In addition, the assumption of linearity upon small bias implies that the magnitude of the perturbation can be set arbitrarily on $\Gamma_{1}$ and $\Gamma_{4}$ (see Appendix 2). It is further assumed that the MIEC|metal interface $\left(\Gamma_{4}\right)$ is blocking the ionic species:

$$
\left.\frac{\partial \tilde{\mu}_{\text {ion }}^{*(1)}}{\partial y}\right|_{\Gamma_{4}}=0
$$


Evaluating this derivative (i.e. differentiating eqn (20)) for the ionic species, and applying the electroneutrality condition (justified by the assumption that electron transfer is facile across the interface), gives, with some rearrangement

$$
\left.\frac{\partial c_{\mathrm{eon}}^{*(1)}}{\partial y}\right|_{\Gamma_{4}}=-\left.4 \frac{c_{\mathrm{ion}}^{\mathrm{eq}}}{c_{\mathrm{eon}}^{\mathrm{eq}}} \frac{\partial \phi^{*(1)}}{\partial y}\right|_{\Gamma_{4}}
$$

Turning to the $\Gamma_{2}$ and $\Gamma_{3}$ boundaries, it follows from symmetry that there is no change in electrochemical potentials for either ions or electrons (and therefore no change in $c_{\text {eon }}^{*(1)}$ or $\left.\phi^{*(1)}\right)$ across the boundaries between each repeating domain.

The next boundary of interest is that at the MIEC|gas interface $\left(\Gamma_{5}\right)$. It is assumed that, as in the bulk, the interfacial reaction behaves linearly upon a small bias. The reaction rate, or equivalently, the current flux, is thus taken to be proportional to the step-change in the electrochemical potential across the interface. For the case of oxygen vacancy (ion) transfer:

$$
\left.\mathbf{j}_{\text {ion }}^{\text {chg }} \cdot \hat{\mathbf{y}}\right|_{\Gamma_{5}}=-k\left(\left.\tilde{\mu}_{\text {ion }}^{*(1)}\right|_{\Gamma_{5+}}-\left.\tilde{\mu}_{\text {ion }}^{*(1)}\right|_{\Gamma_{5}}\right)
$$

with

$$
k=\tilde{R}_{\mathrm{rxn}}^{*-1}
$$

where $k$ is the surface reaction rate-constant and $\tilde{R}_{\text {rxn }}^{*}$ is the area-specific reaction resistance, $\Gamma_{5}+$ denotes the exposed surface of the MIEC, $\Gamma_{5}$ denotes the MIEC immediately beneath the surface, and $\hat{\mathbf{y}}$ is a unit vector in the direction perpendicular to the interface. This is essentially the ChangJaffe boundary condition. ${ }^{38}$ With the assumption that electron transfer across the MIEC/gas interface is rapid, and that there is no gas-phase concentration gradient outside of the MIEC, eqn (26) can be rewritten as (see Appendix 1 for details):

$$
\left.\mathbf{j}_{\text {ion }}^{\text {chg }} \cdot \hat{\mathbf{y}}\right|_{\Gamma_{5}}=-k\left(\left.\tilde{\mu}_{\text {eon }}^{*(1)}\right|_{\Gamma_{5}}-\left.\tilde{\mu}_{\text {ion }}^{*(1)}\right|_{\Gamma_{5}}\right)
$$

\subsection{Numerical method}

Numerical solutions to the differential equations and boundary conditions (summarized in Appendix 2) were pursued using an h-adapted, finite element method (FEM) on an anisotropic triangular mesh. ${ }^{39}$ The equations were discretized on a triangular conforming mesh, using quadratic lagrangian basis functions with a third order bubble, and then solved using the free and open source package FreeFem++. ${ }^{40}$ The mesh was adaptively refined up to seven times at each solution step according to the method illustrated in previous work. ${ }^{12}$ The h-adaptation ensures high regularity of the $H^{1}$ estimator, locally below $0.01 \%$, and it guarantees that the mesh is finer where the sharpest gradients occur. Accordingly, the mesh adaptivity results in coarseness everywhere except in the vicinity of the interfaces (Fig. 2); in particular, the refinement increases as the triple-phase boundary is approached. Integral tests were also implemented in order to ensure that at each iterated solution the numerical method is consistent with the boundary conditions and that it satisfies global conservation of charge. Finally it should be noted that FreeFem ++ execution time is comparable to custom-written $\mathrm{C}++$ code. Due to the sparsity of the problem and its relatively small size
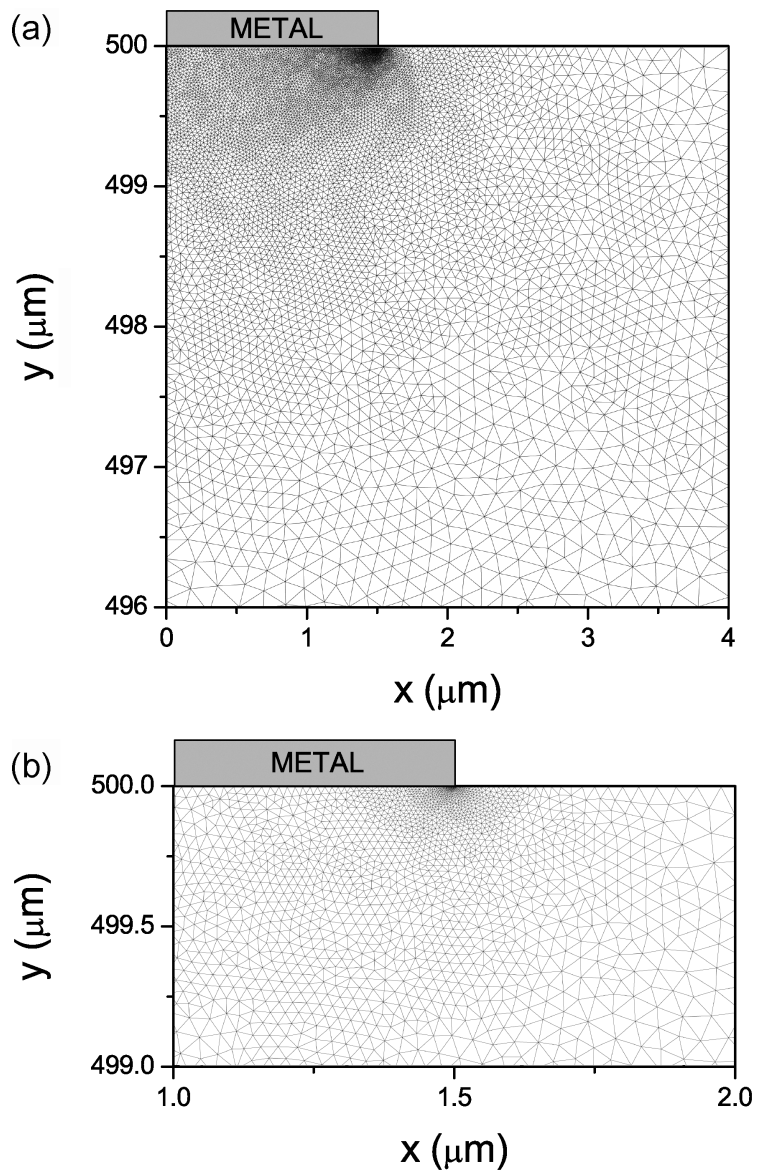

Fig. 2 Example of a refined mesh-grid used for the numerical finiteelement solution. (b) A zoomed-in view of (a).

(typically involving less than $2 \times 10^{5}$ degrees of freedom), the fast direct linear solver, UMFPACK, ${ }^{41}$ was utilized to reduce the solution time.

\subsection{Parameters}

Diffusion coefficients, along with carrier formation energies, have been measured for both oxygen vacancies and polarons in SDC1 5 by Lai and Haile ${ }^{23}$ using electrochemical impedance spectroscopy (Table 1). The measurements span a wide range of temperatures $\left(500-650{ }^{\circ} \mathrm{C}\right)$ and oxygen partial pressures $\left(10^{-31}\right.$ to $\left.0.21 \mathrm{~atm}\right)$, typical for intermediate-temperature SOFC components. The carrier concentrations agree with the dilute-solution defect model described earlier, and the carrier formation energies are consistent with independent, thermogravimetry measurements. ${ }^{33}$ Turning to the remaining parameter, the surface reaction rate-constant (describing hydrogen electro-oxidation over ceria surface), we use the $p_{\mathrm{O}_{2}}$-dependent rate law reported by Lai and Haile ${ }^{23}$ and by Chueh et al. ${ }^{30} \mathrm{We}$ express the rate-constant as:

$$
k=k^{0} p_{\mathrm{O}_{2}}^{-1 / 4}
$$

where $k^{0}$ is the $p_{\mathrm{O}_{2}}$-independent rate-constant. Because the absolute value of $k^{0}$ depends on surface microstructure and cannot be readily extracted from typical experiments, a range of discrete values of $k^{0}$ were utilized in the calculations. 
Table 1 Simulation parameters for porous metal|SDC15|porous metal symmetric cell in $\mathrm{H}_{2}-\mathrm{H}_{2} \mathrm{O}-\mathrm{Ar}$. Values inside parentheses indicate range examined

\begin{tabular}{lll}
\hline Parameter & Value & Unit \\
\hline$T$ & 650 & ${ }^{\circ} \mathrm{C}$ \\
$p_{\mathrm{O}_{2}}$ & $\left(2 \times 10^{-21}-4 \times 10^{-26}\right)$ & $\mathrm{atm}$ \\
$D_{\text {ion }}$ & $1.96 \times 10^{-10}$ & $\mathrm{~m}^{2} \mathrm{~s}^{-1}$ \\
$D_{\text {eon }}$ & $6.46 \times 10^{-9}$ & $\mathrm{~m}^{2} \mathrm{~s}^{-1}$ \\
$\Delta G_{\text {red }}^{0}$ & 3.09 & $\mathrm{eV}$ \\
$\Delta G_{g}^{0}$ & -4.08 & $\mathrm{eV}$ \\
$c_{\text {dop }}$ & $3.75 \times 10^{27}$ & $\mathrm{~m}^{-3}$ \\
$W_{1}$ & $1.5(0.5$ to 3$)$ & $\mu \mathrm{m}$ \\
$W_{2}$ & $2.5(0.1$ to 15$)$ & $\mu \mathrm{m}$ \\
$l$ & 500 & $\mu \mathrm{m}$ \\
$k^{0}$ & $\left(6 \times 10^{-3}-6 \times 10^{-8}\right)$ & $\Omega^{-1} \mathrm{~cm}^{-2} \mathrm{~atm}^{\frac{1}{4}}$ \\
\end{tabular}

In general, the effects of surface reaction rate, oxygen partial pressure and current collector pattern dimension on carrier distribution and equipotential and flux lines (for both ionic and electronic carriers) were explored. The bulk parameters were, in all cases, held fixed as those listed in Table 1.

\section{Results}

\subsection{Analysis quantities: resistances and the} 'surface-influence-zone'

The computed iso-electrochemical potential and flux lines for oxygen vacancies and electrons are presented in Fig. 3 for a typical set of conditions $\left(T=650{ }^{\circ} \mathrm{C}, p_{\mathrm{O}_{2}}=4.1 \times 10^{-26}\right.$ atm, at which the electronic transference number is 0.86 ) and three representative reaction rate-constants: $k^{0}=3 \times 10^{-7}, 6 \times 10^{-7}$, and $\left.3 \times 10^{-5} \Omega^{-1} \mathrm{~cm}^{-2} \mathrm{~atm}^{\frac{1}{4}}\right)$. While the calculation is explicitly carried out for a voltage perturbation of $1 \mathrm{~V}$ between $\Gamma_{1}$ and $\Gamma_{4}$, the assumption of linearity, which applies to small perturbations, implies that the relative values of the electrochemical potentials are more meaningful than their absolute values. In general, the oxygen vacancy equi-potential and flux lines are linear through the bulk of the cell. In the vicinity of the termination of the MIEC, these lines bend gently around the vacancy-blocking MIEC $\mid$ metal interface $\left(\Gamma_{4}\right)$ (Fig. 3(b,d,f)). In contrast, the equi-potential and flux lines for electrons are sharply non-linear even at some distance removed from the termination of the MIEC (Fig. 3(a,c,e)). Moreover, the plots clearly reveal that, in addition to a flow of electronic current between the electrodes on opposing sides of the cell, electronic current flows laterally between the metal current collector $\left(\Gamma_{4}\right)$ and the MIEC|gas $\left(\Gamma_{5}\right)$ surface.

To establish the resistance terms that characterize the electrode interfacial impedance, it is necessary to describe and quantify these various currents. Current that crosses $\Gamma_{1}$, in direct response to an applied bias across the metal current collector at $\Gamma_{4}$, is termed cross-plane current and is denoted by the superscript ' $\mathrm{CP}$ '; that which does not cross $\Gamma_{1}$ is termed in-plane current and denoted by 'IP'. Because of the vacancyblocking behavior of the MIEC|metal interface, the entirety of the ionic current is cross-plane in nature. In contrast, both cross-plane and in-plane electronic current exist, as already noted, where the in-plane electronic current is generated as a result of the electrochemical reactions. The magnitude of the in-plane electronic current is precisely equal to the ionic
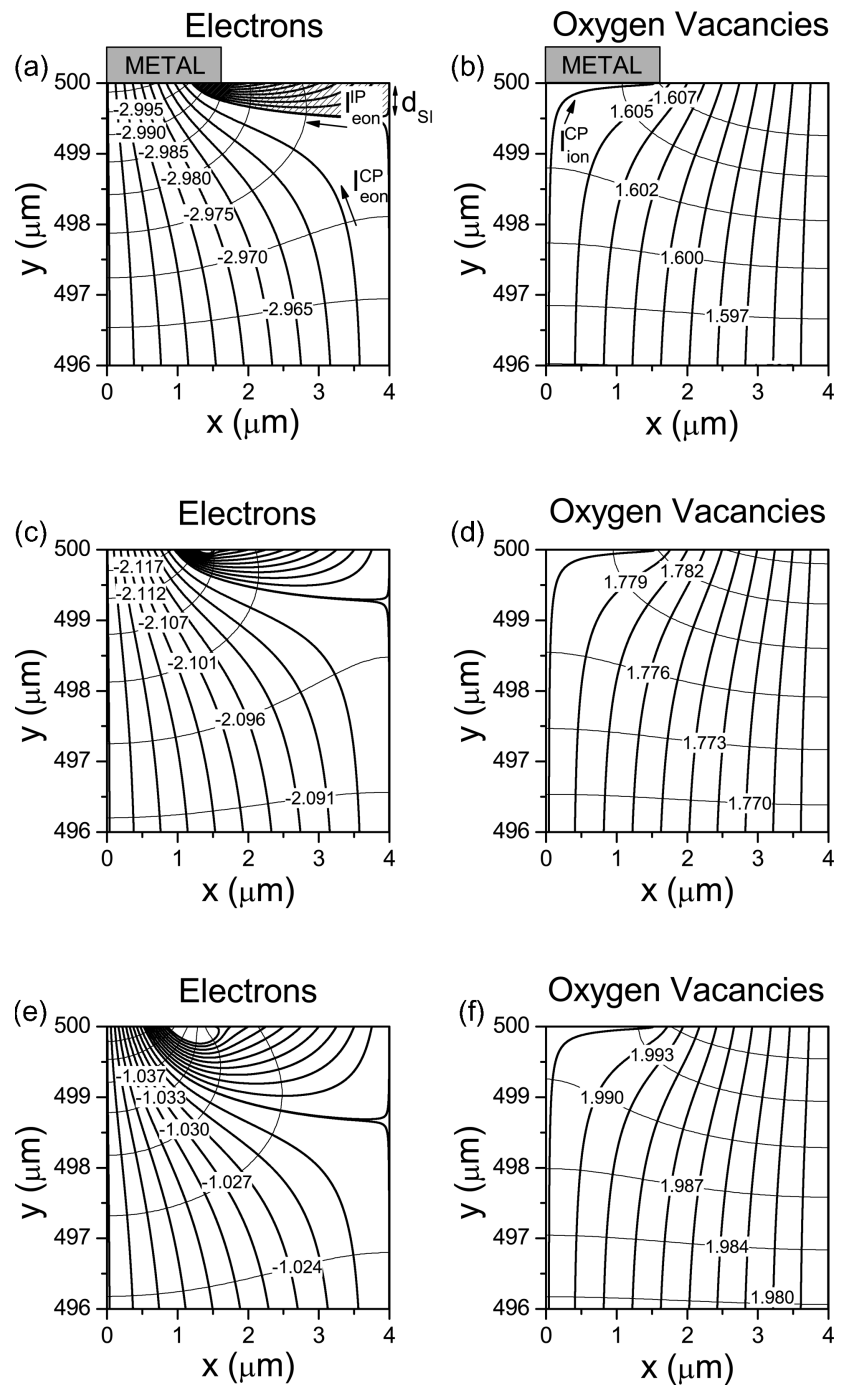

Fig. 3 Electronic and ionic electrochemical equi-potential lines (thin) and the corresponding current flux lines (thick) computed at $T=650{ }^{\circ} \mathrm{C}$ and $p_{\mathrm{O}_{2}}=4.1 \times 10^{-26}$ atm for (a-b) $k^{0}=3 \times 10^{-7}$, (c-d) $k^{0}=6 \times 10^{-7}$, and $(\mathrm{e}-\mathrm{f}) k^{0}=3 \times 10^{-5} \Omega^{-1} \mathrm{~cm}^{-2} \mathrm{~atm}^{\frac{1}{4}}$. Only the region close to the interface is shown; the potentials and fluxes are essentially linear beyond the region plotted. Hatched area in (a) is the surface-influence-zone.

cross-plane current, in accord with the global electrochemical reaction, eqn (1). The charge flow associated with each of these three currents is explicitly indicated in Fig. 3(a) and 3(b). Noting that current is simply the integrated flux over a given area, the following formal definitions and relationships apply to these currents:

$$
\begin{aligned}
& I_{\text {ion }}^{\mathrm{CP}}=\int_{\Gamma_{1}} \mathrm{j}_{\mathrm{ion}}^{\mathrm{chg}} \cdot \hat{\mathbf{y}} \mathrm{d} x=\int_{\Gamma_{5}} \mathrm{j}_{\mathrm{ion}}^{\mathrm{chg}} \cdot \hat{\mathbf{y}} \mathrm{d} x \\
& I_{\mathrm{eon}}^{\mathrm{IP}}=\int_{\Gamma_{5}} \mathrm{j}_{\mathrm{eon}}^{\mathrm{chg}} \cdot \hat{\mathbf{y}} \mathrm{d} x \\
& I_{\mathrm{eon}}^{\mathrm{CP}}=\int_{\Gamma_{1}} \mathrm{j}_{\mathrm{eon}}^{\mathrm{chg}} \cdot \hat{\mathbf{y}} \mathrm{d} x \\
& I_{\mathrm{ion}}^{\mathrm{CP}}=-I_{\mathrm{eon}}^{\mathrm{IP}} \\
& I_{\text {ion }}^{\mathrm{IP}}=0
\end{aligned}
$$




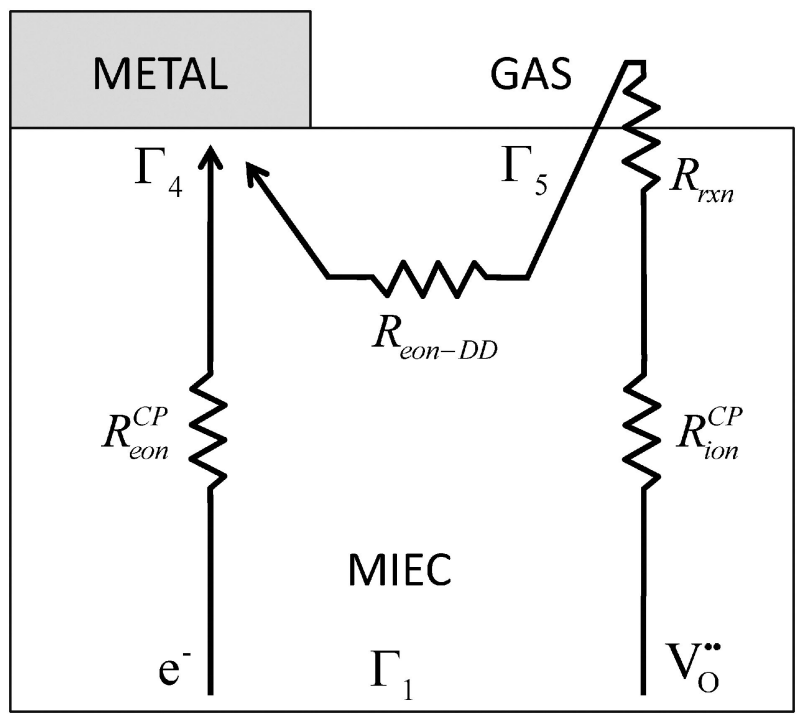

Fig. 4 Schematic of the various current and corresponding resistances in the MIEC. Arrows indicate direction of the charge flux.

An experimental measurement of the total resistance across the entire electrochemical cell corresponds to a measurement of the ratio of the perturbation of the electrochemical potential of electrons on the $\Gamma_{4}$ surface to the total electronic and ionic current:

$$
R_{\mathrm{TOT}}=\left.\tilde{\mu}_{\mathrm{eon}}^{*(1)}\right|_{\Gamma_{4}} /\left(I_{\mathrm{eon}}^{\mathrm{CP}}+I_{\mathrm{ion}}^{\mathrm{CP}}\right)
$$

Several individual resistive steps contribute to this total cell resistance. They can be numerically isolated by inserting the flux definitions given above with the electrochemical potential drops that occur at various positions within the cell, Fig. 4. Four resistances are identified: the cross-plane bulk electronic resistance, $R_{\mathrm{eon}}^{\mathrm{CP}}$, the cross-plane bulk ionic resistance, $R_{\mathrm{ion}}^{\mathrm{CP}}$, the in-plane electron diffusion-drift resistance, $R_{\text {eon-DD, }}$, and the surface reaction resistance, $R_{\text {rxn }}$. From Fig. 4 , we can see that $R_{\text {rxn }}, R_{\text {eon-DD }}$, and $R_{\text {ion }}^{\mathrm{CP}}$ are connected in series because these terms correspond to the electrochemical reaction, surface and bulk diffusions necessary to form and transport oxygen vacancies across the electrochemical cell. In parallel with the transport of oxygen vacancies is the transport of electrons across the cell. Thus, the total resistance can be written as:

$$
R_{\mathrm{TOT}}=\left(\left(R_{\mathrm{eon}-\mathrm{DD}}+R_{\mathrm{rxn}}+R_{\mathrm{ion}}^{\mathrm{CP}}\right)^{-1}+\left(R_{\mathrm{eon}}^{\mathrm{CP}}\right)^{-1}\right)^{-1}
$$

with

$$
\begin{aligned}
R_{\mathrm{ion}}^{\mathrm{CP}} & =\frac{\left\langle\tilde{\mu}_{\mathrm{ion}}^{*}\right\rangle_{\Gamma_{1}}-\left\langle\tilde{\mu}_{\mathrm{ion}}^{*}\right\rangle_{\Gamma_{5}}}{I_{\mathrm{ion}}^{\mathrm{CP}}} \\
R_{\mathrm{eon}}^{\mathrm{CP}} & =\frac{\left\langle\tilde{\mu}_{\mathrm{eon}}^{*}\right\rangle_{\Gamma_{1}}-\left\langle\tilde{\mu}_{\mathrm{eon}}^{*}\right\rangle_{\Gamma_{4}}}{I_{\mathrm{eon}}^{\mathrm{CP}}} \\
R_{\mathrm{eon}-\mathrm{DD}} & =\frac{\left\langle\tilde{\mu}_{\mathrm{eon}}^{*}\right\rangle_{\Gamma_{4}}-\left\langle\tilde{\mu}_{\mathrm{eon}}^{*}\right\rangle_{\Gamma_{5}}}{I_{\mathrm{eon}}^{\mathrm{IP}}} \\
R_{\mathrm{rxn}} & =\frac{\left\langle\tilde{\mu}_{\mathrm{ion}}^{*}\right\rangle_{\Gamma_{5}}-\left\langle\tilde{\mu}_{\mathrm{eon}}^{*}\right\rangle_{\Gamma_{5}}}{I_{\mathrm{ion}}^{\mathrm{CP}}}
\end{aligned}
$$

where the brackets indicate averaging over the specified interface. (Note: eqn (39) involves a subtle approximation in which electrons are taken to migrate between the entirety of the MIEC|metal interface and the MIEC|gas interface. For details, see Appendix 3.).

The total electrode resistance, $R_{\text {ion }}^{\perp}$, is readily recognized from Fig. 4 as the sum of $R_{\text {rxn }}$ and $R_{\text {eon-DD }}$. Combining eqn (33), (39) and (40) this becomes:

$$
R_{\mathrm{ion}}^{\perp}=\frac{\left\langle\tilde{\mu}_{\mathrm{ion}}^{*}\right\rangle_{\Gamma_{5}}-\left\langle\tilde{\mu}_{\mathrm{eon}}^{*}\right\rangle_{\Gamma_{4}}}{I_{\mathrm{ion}}^{\mathrm{CP}}}=R_{\mathrm{rxn}}+R_{\mathrm{eon}-\mathrm{DD}}
$$

The proportion of the electrode resistance due to the surface reaction is simply:

$$
f_{\mathrm{rxn}}=\frac{R_{\mathrm{rxn}}}{R_{\mathrm{rxn}}+R_{\mathrm{eon}-\mathrm{DD}}}=\frac{R_{\mathrm{rxn}}}{R_{\mathrm{ion}}^{\perp}}
$$

where $f_{\text {rxn }}$ is termed the fractional surface resistance. The conventional, area-specific interfacial resistance, $\tilde{R}$, is given by a normalization of the resistance terms relative to the macroscopic electrode area, $W_{1}+W_{2}$ (for unit thickness). Normalization with respect to the active area, $W_{2}$, is denoted here as $\tilde{R}^{*}$.

In addition to interfacial resistance, it is valuable to consider the features of the surface-electron penetration into the MIEC as a result of the interaction between the in-plane and cross-plane electronic current. Fig. 3(a, c and e) reveal that electrons injected/removed from the surface reaction site furthest from the metal current collector (the intersection of $\Gamma_{3}$ and $\Gamma_{5}$ in Fig. 1(b)) follow a trajectory of maximum penetration, defining the boundary of the surface-influencezone. Within this region, the entirety of the electronic current flows in the in-plane direction. The maximum penetration depth of the surface-influence-zone, $d_{\text {SIZ }}$, is used hereafter to quantify the magnitude of this region.

\subsection{Influence of surface reaction rate and oxygen partial pressure}

The computed equi-potential and flux lines of Fig. 3 reveal that the oxygen vacancy behavior depends very weakly on the surface reaction rate-constant. The same is true of their dependence on oxygen partial pressure (not shown). In contrast, the electron equi-potential and flux lines depend strongly on both the magnitude of the surface reaction rate-constant and the oxygen partial pressure. Accordingly, the penetration depth of the surface-reaction-zone, $d_{\text {SIZ }}$, also varies strongly with $p_{\mathrm{O}_{2}}$ and $k^{0}$, spanning a wide range of values from less than 0.1 to more than $4 \mu \mathrm{m}$ under the conditions explored, Fig. 5. The manner in which these terms establish $d_{\text {SIZ }}$ can be understood from an evaluation of the relative magnitudes of the in-plane and cross-plane electronic currents, also presented in Fig. 5. It is immediately evident that the two derived quantities trend in almost an identical manner with the two input variables. This behavior results because the penetration depth, Fig. 3, reflects the position at which the two types of electronic flux attain a balance within the volume of the MIEC. For this reason, the ratio $I_{\mathrm{eon}}^{\mathrm{IP}} / I_{\mathrm{eon}}^{\mathrm{CP}}$ directly sets the magnitude of the surface-influence-zone. 
(a)

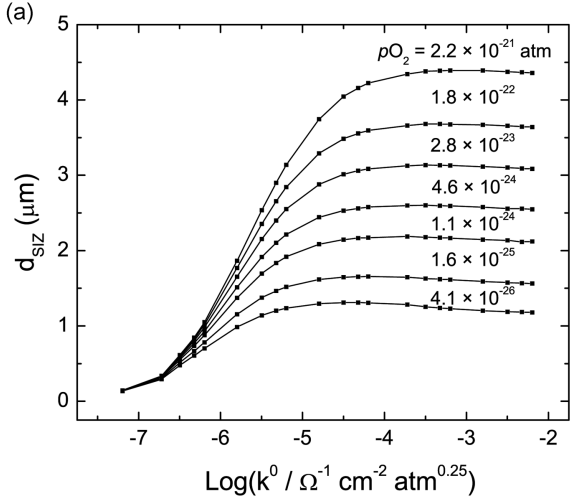

(c)

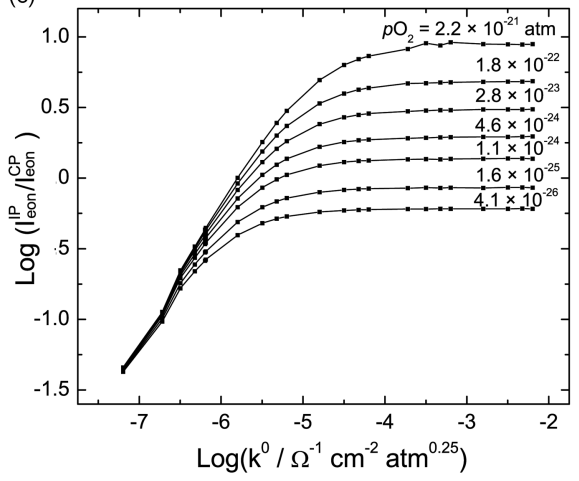

$\log \left(\sigma_{\text {eon }} / \Omega^{-1} \mathrm{~cm}^{-1}\right)$

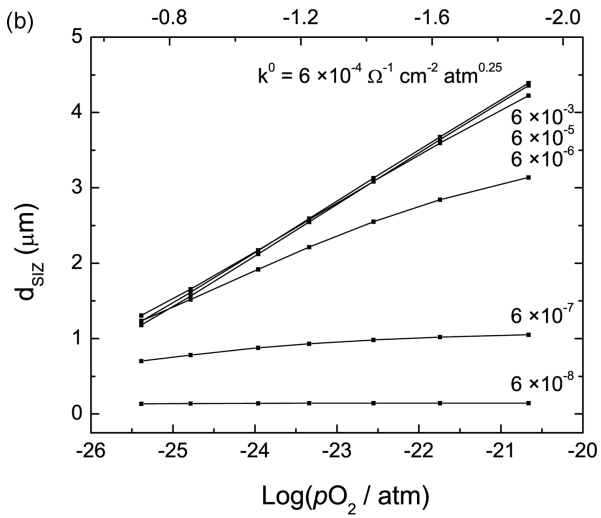

$\log \left(\sigma_{\text {eon }} / \Omega^{-1} \mathrm{~cm}^{-1}\right)$

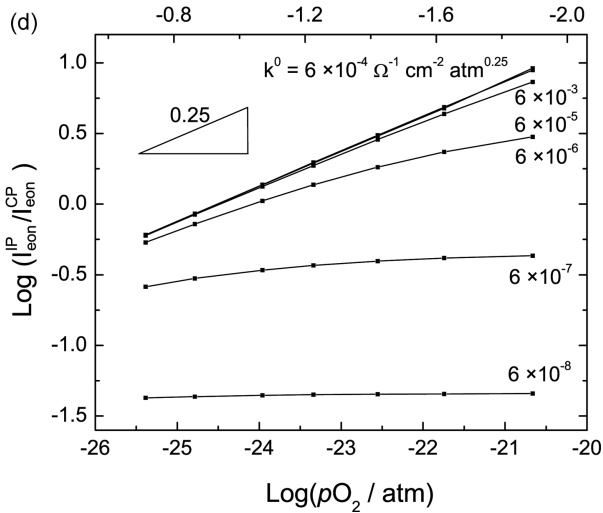

Fig. 5 (a, b) Penetration-depth of the surface-influence-zone at various surface reaction rate-constants and oxygen partial pressures. (c, d) Ratio of the in-plane to the cross-plane electronic current. $T=650^{\circ} \mathrm{C}$ for all plots. The bulk electronic conductivity for SDC15 is also shown for plots (b) and (d).

The results summarized in Fig. 5 reveal that, at fixed oxygen partial pressure, the ratio of the currents (and hence $d_{\mathrm{SIZ}}$ ) generally increases with $k^{0}$, but eventually reaches a limiting value beyond which further increases in reaction rate have no impact. The dependence on $p_{\mathrm{O}}$ at fixed $k^{0}$ is somewhat more subtle. When $k^{0}$ is small, $I_{\mathrm{eon}}^{\mathrm{IP}} / I_{\mathrm{eon}}^{\mathrm{CP}}$ (and $d_{\mathrm{SIZ}}$ ) are independent of $p_{\mathrm{O}_{2}}$, but when $k^{0}$ is large, they display a $p_{\mathrm{O}_{2}}^{1 / 4}$ dependence. These trends can be understood as follows. Because the in-plane electron current is equal in magnitude to the crossplane ionic current (eqn (33) and Fig. 4), $I_{\text {eon }}^{\mathrm{IP}} / /_{\text {eon }}^{\mathrm{CP}}$ can be written as

$$
\left|\frac{I_{\text {eon }}^{\mathrm{IP}}}{I_{\mathrm{eon}}^{\mathrm{CP}}}\right|=\left|\frac{I_{\mathrm{ion}}^{\mathrm{CP}}}{I_{\mathrm{eon}} \mathrm{CP}}\right|=\frac{R_{\mathrm{eon}}^{\mathrm{CP}}}{R_{\mathrm{ion}}^{\mathrm{CP}}+R_{\mathrm{rxn}}+R_{\mathrm{eon}-\mathrm{DD}}}
$$

Essentially, $I_{\mathrm{eon}}^{\mathrm{IP}} / I_{\mathrm{eon}}^{\mathrm{CP}}$ represents the relative contributions of the two parallel current paths shown in Fig. 4.

For very low values of $k^{0}$, the surface reaction resistance dominates the denominator, and the above ratio becomes:

$$
\left|\frac{I_{\mathrm{eon}}^{\mathrm{IP}}}{I_{\mathrm{eon}}^{\mathrm{CP}}}\right| \approx \frac{R_{\mathrm{eon}}^{\mathrm{CP}}}{R_{\mathrm{rxn}}}
$$

In this limit, $I_{\mathrm{eon}}^{\mathrm{IP}} / I_{\mathrm{eon}}^{\mathrm{CP}}$ is approximately proportional to $k$ (eqn (29) and, hence, at fixed $p_{\mathrm{O}_{2}}$ this ratio and $d_{\mathrm{SIZ}}$ increase monotonically with $k^{0}$. In terms of the dependence on oxygen partial pressure both $R_{\mathrm{rxn}}$ and $R_{\mathrm{eon}}^{\mathrm{CP}}$ scale with $p_{\mathrm{O}_{2}}^{1 / 4}$, and thus $I_{\mathrm{eon}}^{\mathrm{IP}} / I_{\mathrm{eon}}^{\mathrm{CP}}$ and $d_{\mathrm{SIZ}}$ do not depend on $p_{\mathrm{O}}$.

In the limit of very large $k^{0}$, the surface reaction resistance tends to zero, and eqn (43) becomes:

$$
\left|\frac{I_{\mathrm{eon}}^{\mathrm{IP}}}{I_{\mathrm{eon}}^{\mathrm{CP}}}\right| \approx \frac{R_{\mathrm{eon}}^{\mathrm{CP}}}{R_{\mathrm{ion}}^{\mathrm{CP}}+R_{\mathrm{eon}-\mathrm{DD}}} \approx \frac{R_{\mathrm{eon}}^{\mathrm{CP}}}{R_{\mathrm{ion}}^{\mathrm{CP}}}
$$

The elimination of $R_{\mathrm{rxn}}$ from this expression implies both $I_{\mathrm{eon}}^{\mathrm{IP}} / I_{\mathrm{eon}}^{\mathrm{CP}}$ and $d_{\mathrm{SIZ}}$ become independent of $k^{0}$, as is observed. Physically, this corresponds to a situation in which the reaction rate is so fast that the rate at which electrons can be injected/removed at the MIEC/gas surface exceeds the rate at which they are removed by diffusion-drift and hence the reaction rate no longer has any influence on the system behavior. Under the simulation conditions used in this work, $R_{\mathrm{ion}}^{\mathrm{CP}} \gg R_{\text {eon-DD }}$ and the role of $R_{\text {eon-DD }}$ is also minimal in setting the behavior, as indicated in the right-side simplification to eqn (45). Hence, at fixed (large) $k^{0}$ the oxygen partial pressure dependence of $I_{\mathrm{eon}}^{\mathrm{IP}} / I_{\mathrm{eon}}^{\mathrm{CP}}$ (and of $d_{\mathrm{SIZ}}$ ) is largely established by the properties of $R_{\mathrm{eon}}^{\mathrm{CP}}$. As $R_{\mathrm{eon}}^{\mathrm{CP}}$ scales with $p_{\mathrm{O}_{2}}^{1 / 4}$, so do $I_{\mathrm{eon}}^{\mathrm{IP}} / I_{\mathrm{eon}}^{\mathrm{CP}}$ and $d_{\mathrm{SIZ}}$. This combination of trends implies that the largest penetration of the surface-influence zone occurs at large $k^{0}$ and, counter-intuitively, high $p_{\mathrm{O}_{2}}$ (under which the surface injection current is low). At these extremes, which 

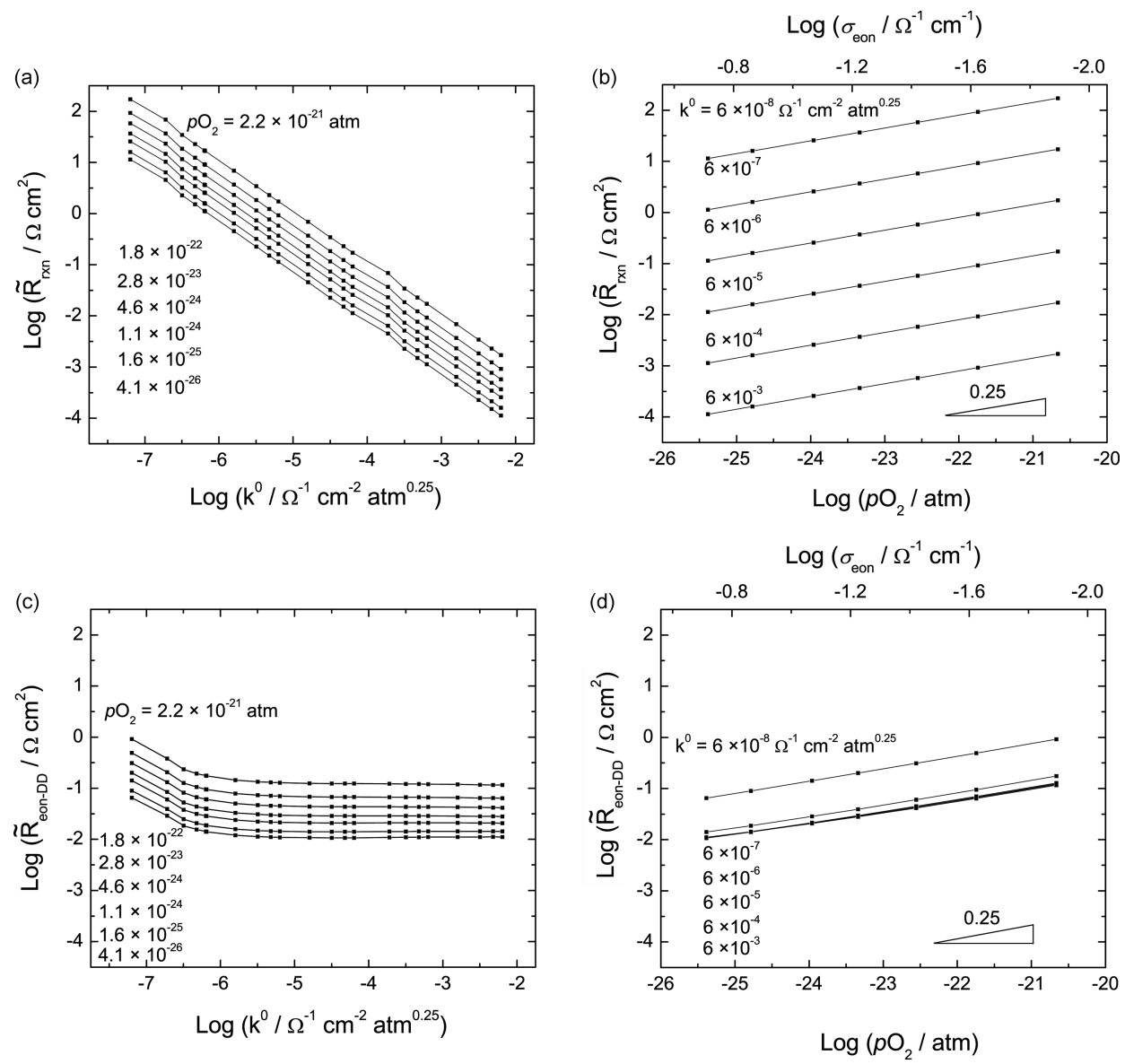

Fig. 6 (a,b) The area-specific surface reaction resistance (normalized by the total surface area, $\left.W_{1}+W_{2}\right), \tilde{R}_{\mathrm{rxn}}$ and (c,d) the area specific diffusion-drift resistance, similarly normalized, $\tilde{R}_{\text {eon-DD }}$ as a function of surface reaction rate-constant and oxygen partial pressure at $T=650{ }^{\circ} \mathrm{C}$. The bulk electronic conductivity for SDC15 is also shown for plots (b) and (d).

correspond to plausible physical conditions and material properties, the penetration depth exceeds the width of the MIEC $\mid$ gas interface.

The explicit influences of oxygen partial and reaction rateconstant on the interfacial resistance terms, $R_{\text {eon-DD }}$ and $R_{\text {rxn }}$, computed according to eqn (39) and (40), respectively, are presented in Fig. 6. By definition, eqn (27) and (29), the surface reaction resistance scales inversely with $k^{0}$ and scales linearly with $p_{\mathrm{O}_{2}}^{1 / 4}$, Fig. 6(a and b). The in-plane electron diffusion-drift resistance displays a similar $p_{\mathrm{O}_{2}}^{1 / 4}$-dependence, Fig. 6(d), in this case due to the nature of the dependence of electronic resistivity on oxygen partial pressure, also shown in the figure. In contrast to $R_{\text {rxn }}$, however, $R_{\text {eon-DD }}$, decreases then plateaus with increasing reaction rate-constant, mirroring to some extent the behavior of $d_{\text {SIZ }}$, Fig. 6(c). The limiting behavior as $k^{0}$ increases simply indicates that beyond some reaction rate, the discontinuity in oxygen vacancy electrochemical potential at the $\Gamma_{5}$ boundary, eqn (26), approaches zero (i.e. $\left\langle\tilde{\mu}_{\mathrm{ion}}^{*}\right\rangle_{\Gamma_{4}}=\left\langle\tilde{\mu}_{\mathrm{eon}}^{*}\right\rangle_{\Gamma_{5}}$ ) and the solutions (i.e. equi-potential and flux lines) no longer depend on reaction rate, as noted above. If one considers the behavior of the overall interfacial impedance term, $R_{\mathrm{ion}}^{\perp}$, (the sum of the reaction and electron diffusion-drift terms, eqn (41)), because both component terms depend on oxygen partial pressure according to $p_{\mathrm{O}_{2}}^{1 / 4}, R_{\text {ion }}^{\perp}$ will also scale with $p_{\mathrm{O}_{2}}^{1 / 4}$ (Fig. 7). Again, this is an immediate consequence of the selected rate law, eqn (29). With respect to variations in $k^{0}$, limiting behavior also occurs for $R_{\mathrm{ion}}^{\perp}$ as it does for other quantities, consistent with the behavior of the system overall. Specifically, as $k^{0}$ increases, the surface reaction resistance becomes negligible and $R_{\mathrm{ion}}^{\perp}$ approaches $R_{\text {eon-DD }}$ (eqn (41)), where the latter is, in general, a finite quantity. The relative contributions of $R_{\text {rxn }}$ and $R_{\text {eon-DD }}$ to $R_{\mathrm{ion}}^{\perp}$ as a function of $p_{\mathrm{O}_{2}}$ and $k^{0}$ are immediately revealed from a plot of $f_{\text {rxn }}$ (the ratio of the $R_{\text {rxn }}$ to $R_{\text {ion }}^{\perp}$ ) as a function of these two quantities, Fig. 8. As expected, when the surface reaction is very fast, the electrode resistance is dominated by in-plane electron diffusion-drift resistance $\left(f_{\text {rxn }}\right.$ approaches zero). On the other hand, when the surface reaction is very slow, the electrode resistance is dominated by the surface reaction resistance ( $f_{\text {rxn }}$ approaches unity). The relative contributions of $R_{\text {eon-DD }}$ and $R_{\text {rxn }}$ to $R_{\text {ion }}^{\perp}$, are largely independent of oxygen partial pressure, as the surface reaction resistance and the in-plane resistance scale with pressure in the same way.

In an one-dimensional analog to the present problem, whether limiting behavior can be expected is readily predicted from an evaluation of a quantity $(k W) / \sigma$, where $W$ is the diffusion length. Specifically, when $(k W) / \sigma \gg 1$, the system behavior is independent of the reaction rate. In the present problem, the influence of reaction rate similarly decreases as 


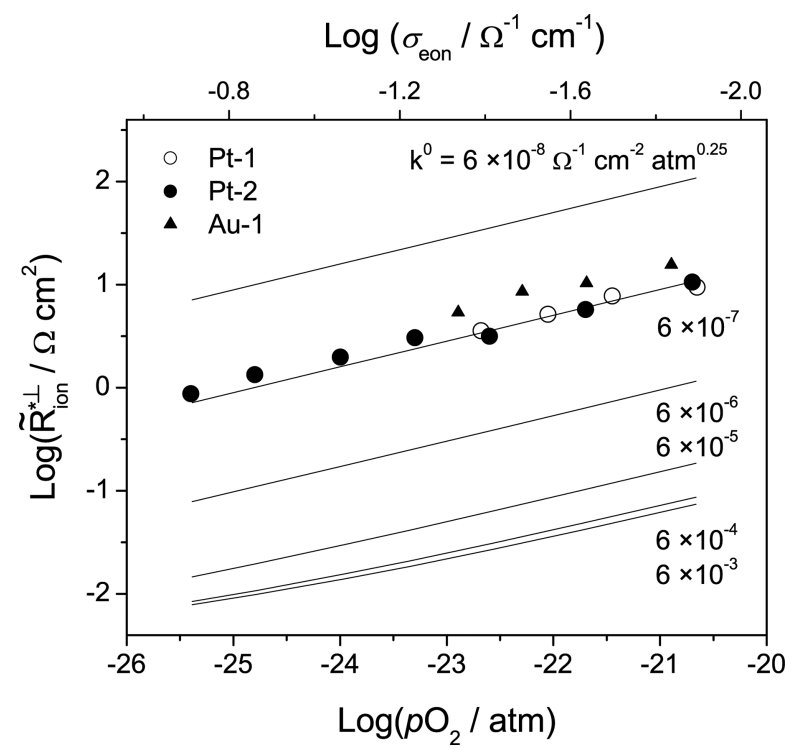

Fig. 7 Area-specific electrode polarization resistance (normalized by the active reaction area, $W_{2}$ ) plotted as a function of oxygen partial pressure and surface reaction rate-constant at $T=650{ }^{\circ} \mathrm{C}$ (lines). Experimental data for SDC15 symmetric cells with Pt (circle) and with $\mathrm{Au}$ (triangle) metal current collectors. (See Table 2 for details of experimental data.)

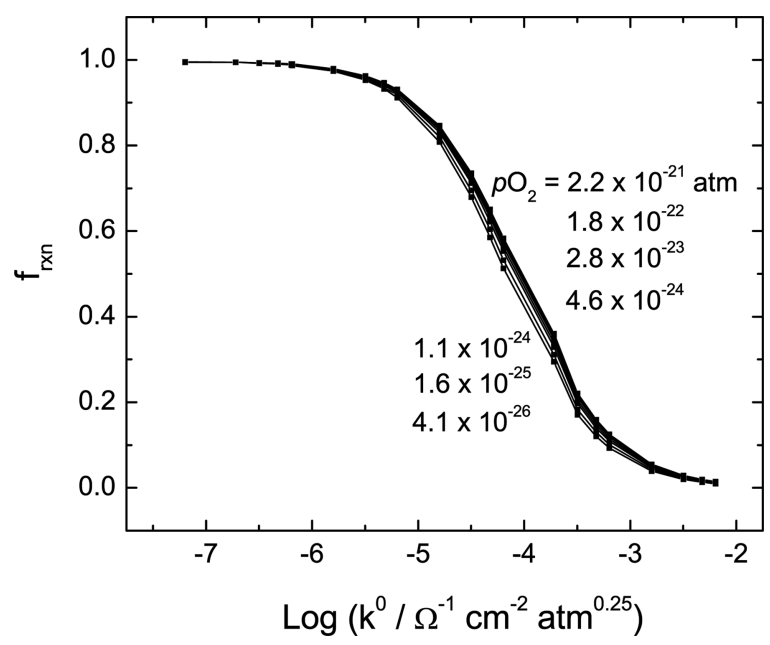

Fig. 8 Fractional surface reaction resistance as a function of surface reaction rate-constant and oxygen partial pressure at $T=650{ }^{\circ} \mathrm{C}$. $f_{\mathrm{rxn}}=1$ indicates that the electrode reaction is limited entirely by the surface-reaction, whereas $f_{\mathrm{rxn}}=0$ indicates that the electrode reaction is limited entirely by in-plane electron diffusion-drift.

$\left(k W_{2}\right) / \sigma_{\text {eon }}$ increases, but a preliminary analysis indicates that the solutions do not scale directly with this dimensionless quantity, primarily due to the fact that the electron penetration-depth is not constant with respect to $k$. While a precise determination of the relevant scaling law is beyond the scope of this study, it is clear that the behavior of the system under consideration here can be approximated by the onedimensional result (with $f_{\text {rxn }}$ being a single-valued function of $\left.\left(k W_{2}\right) / \sigma_{\text {eon }}\right)$ only when $k$ is large, underscoring the importance of a complete, two-dimensional analysis.

\subsection{Geometrical considerations}

The influence of pattern dimensions (at a fixed temperature of $650{ }^{\circ} \mathrm{C}$ and oxygen partial pressure of $p_{\mathrm{O}_{2}}=4.1 \times 10^{-26} \mathrm{~atm}$ ) are captured in a series of contour plots presented in Fig. 9. The geometric degrees of freedom are the metal stripe width $\left(2 W_{1}\right)$ and the inter-metal-stripe distance $\left(2 W_{2}\right)$, represented in the plots by $W_{2} / W_{1}$ and $W_{1}$. Presented in the top row of images is the behavior of the fractional surface resistance, in the middle row is the total electrode resistance normalized by the total electrode area $\left(\tilde{R}_{\text {ion }}^{\perp}\right)$, and in the bottom row the electrode resistance normalized by the active reaction area $\left(\tilde{R}_{\text {ion }}^{* \perp}\right.$, each computed for three values of $k^{0}$.

At relatively low $k^{0}$ (Fig. 9, left column), the interfacial process is dominated by the slow reaction rate and $f_{\mathrm{rxn}}$ remains close to one over the entire range of current collector geometries examined. Under these conditions, the macroscopically measured interfacial resistance, $\tilde{R}_{\text {ion }}^{\perp}$, is independent of the dimensions of the metal current collector, so long as the ratio $W_{2} / W_{1}$, which is a measure of the fraction of the MIEC surface that is available to support the electrochemical reaction, is fixed. Analogously, normalization by the active area gives an interfacial impedance $\tilde{R}_{\text {ion }}^{* \perp}$ that is essentially independent of current collector geometry (varying by less than $6 \%$ over the range of geometries considered). Thus, in the low $k^{0}$ regime, electron diffusion-drift does not contribute to the macroscopic electrode resistance (even for distances as long as $15 \mu \mathrm{m})$. Accordingly, assuming fixed material properties, minimizing the electrode resistance is achieved by maximizing the amount of active surface area available for electrochemical reaction, namely, the MIEC|gas interfacial area.

As the surface reaction rate increases (Fig. 9, middle and right column), so does the influence of current collector geometry on electrode resistance. The fractional surface resistance attains values considerably lower than one and decreases with both increasing $W_{1}$ and $W_{2} / W_{1}$, indicating the electrode resistance is becoming increasingly dominated by in-plane electron diffusion. Consistent with the increased contribution from in-plane diffusion, the macroscopic electrode resistance is no longer constant with respect to the inter-metal distance, as evident in Fig. 9(e) and even more so in Fig. 9(f). Furthermore, at the highest reaction rates $\tilde{R}_{\text {ion }}^{\perp}$ is no longer a monotonic function of $W_{2} / W_{1}$, indicating that the optimal geometry reflects a balance between minimizing diffusion distance and maximizing reaction area. Lastly, the electrode resistance normalized by the active area, $\tilde{R}_{\text {ion }}^{* \perp}$, displays the same trend as the fractional surface resistance, confirming that, in the regime where in-plane diffusion is no longer negligible, current density along the MIEC|gas interface falls as the distance away from the current collector increases.

\subsection{Comparison with experimental results}

Experimental data for the interfacial reaction resistance for hydrogen electro-oxidation on SDC15 are available from earlier measurements by Lai and $\mathrm{Haile}^{23}$ and by Chueh et al. ${ }^{30}$ In those studies, data were collected under $\mathrm{H}_{2}-\mathrm{H}_{2} \mathrm{O}-\mathrm{Ar}$ atmospheres from symmetric $\mathrm{Sm}_{0.15} \mathrm{Ce}_{0.85} \mathrm{O}_{1.925}$ based-cells in which either porous $\mathrm{Pt}$ or porous $\mathrm{Au}$ (of random geometry) 


$$
\mathrm{k}^{0}=3 \times 10^{-7} \Omega^{-1} \mathrm{~cm}^{-2} \mathrm{~atm}^{0.25}
$$
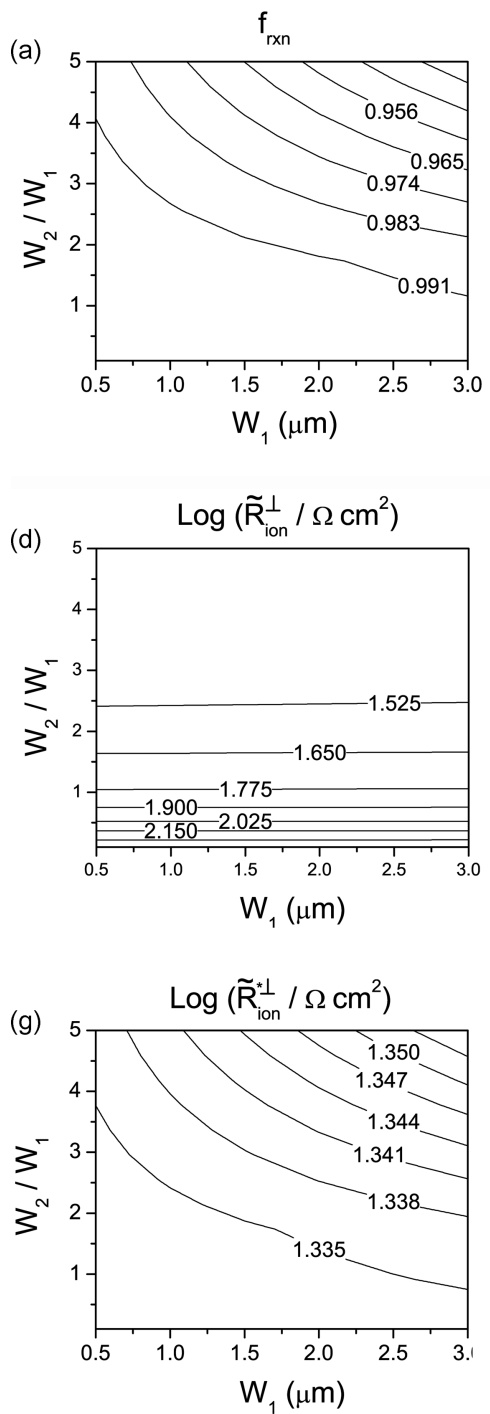

$\mathrm{k}^{0}=3 \times 10^{-6} \Omega^{-1} \mathrm{~cm}^{-2} \mathrm{~atm}^{0.25}$
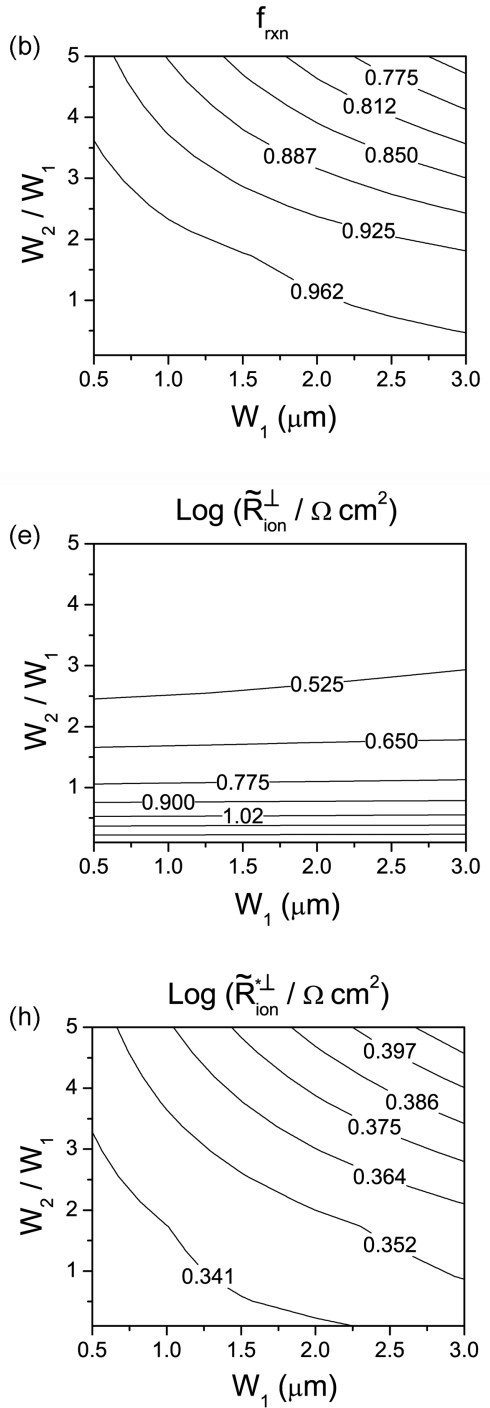

$\mathrm{k}^{0}=3 \times 10^{-5} \Omega^{-1} \mathrm{~cm}^{-2} \mathrm{~atm}^{0.25}$
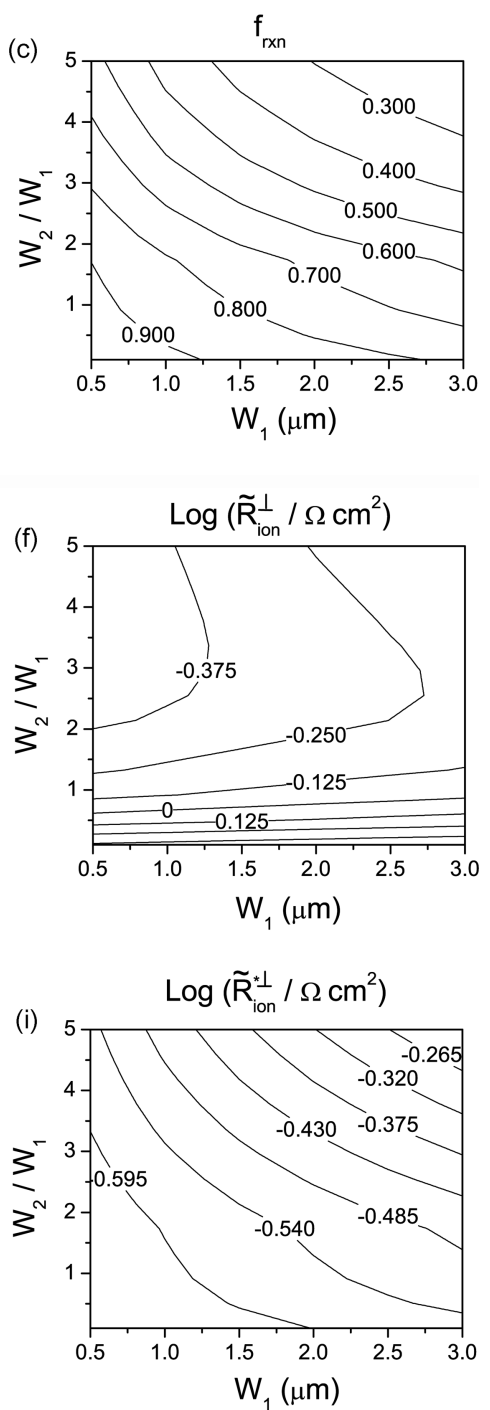

Fig. 9 Contour plots of the fractional surface reaction resistance $\left(f_{\text {rxn }}\right)$ (top row), the area-specific electrode resistance normalized by the total electrode area (middle row), and by the electrode resistance active reaction area (bottom row), as a function of $W_{1}$ and $W_{2}$, the metal current collector stripe width and the distance between stripes, respectively. Each column represents a particular value of the surface reaction rateconstant. $T=650{ }^{\circ} \mathrm{C}$ and $p_{\mathrm{O}_{2}}=4.1 \times 10^{-26} \mathrm{~atm}$.

served as the electrodes. In each case, both bulk and electrode parameters were obtained from the same electrochemical cell, assuring a high-degree of self-consistency. The bulk parameters obtained in the latter study are essentially identical to those listed in Table 1, which explicitly correspond to the earlier study.

To permit a comparison with the computed results presented here, effective values for $W_{1}$ and $W_{2}$ for the random porous electrodes are estimated by equating these geometric parameters to the experimentally-determined average pore size and interpore distance (obtained from microstructural examinations). These values are summarized in Table 2 for two measurements using Pt electrodes (Pt-1 and Pt-2) and one using Au electrodes. For these dimensions and the limiting case of infinitely fast reaction rate, the expected total interfacial resistance, $\tilde{R}_{\text {ion }}^{\perp}$, is of the order of $10^{-2} \Omega \mathrm{cm}^{2}$ at $650{ }^{\circ} \mathrm{C}$ and $1 \times 10^{-25}$ atm oxygen partial pressure. The experimental value of $\tilde{R}_{\text {ion }}^{\perp}$ on the other hand, is approximately two orders of magnitude greater than that, immediately indicating that electron diffusion-drift is not the rate-determining step. For the case in which interfacial reaction resistance is ratelimiting, the appropriate normalization is with respect to the active area $\left(W_{2}\right)$ and, accordingly, the experimental $\tilde{R}_{\text {ion }}^{* \perp}$ values are compared to the calculated values presented in Fig. 7.

The comparison presented in Fig. 7 (for the specific temperature of $650{ }^{\circ} \mathrm{C}$ ) reveals several important features. Most significantly, although the interfacial impedance normalized to macroscopic area differs for the three different measurements by $\sim 3$ times (as reported in the original work), the normalization according to active area yields experimental values that are in relatively good agreement with one another, 
Table 2 Experimentally-determined microstructural parameters for the porous metal $|\mathrm{SDC} 15|$ porous metal electrochemical cells reported in literature

\begin{tabular}{lllll}
\hline Sample & Current collector metal & $W_{1} / \mu \mathrm{m}$ & $W_{2} / \mu \mathrm{m}$ & Ref. \\
\hline $\mathrm{Pt}-1$ & $\mathrm{Pt}$ & 1.5 & 2.5 & 23 \\
$\mathrm{Pt}-2$ & $\mathrm{Pt}$ & 1.5 & 2.5 & 30 \\
$\mathrm{Au}$ & $\mathrm{Au}$ & 6.0 & 3.2 & 30 \\
\hline
\end{tabular}

consistent with a reaction-limited process. Such an agreement, occurring as it does despite the difference in metallic component, suggests that the metal is not involved in the rate-limiting step. Moreover, the experimental interfacial reaction rate-constant clearly obeys a $p_{\mathrm{O}_{2}}^{-1 / 4}$ rate law, although such a result is not a priori expected. In the earlier work it was speculated that this behavior was due to an interfacial process that is limited by electron diffusion-drift, which, by definition, scales with $p_{\mathrm{O}_{2}}^{-1 / 4}$ and is independent of the nature of the metallic current collector. The present calculation, however, indicates that, barring highly unusual electron transport properties along the MIEC|gas interface, electron diffusiondrift is sufficiently rapid that it can be ruled out as the cause of the observed oxygen partial pressure dependence. The corollary of this conclusion is that some other factor, tentatively assigned as the reaction rate, must scale with $p_{\mathrm{O}_{2}}^{-1 / 4}$. Accepting this implication, the experimental data yield a value for $k^{0}$ of approximately $6 \times 10^{-7} \Omega^{-1} \mathrm{~cm}^{-2} \mathrm{~atm}^{\frac{1}{4}}$ (at $T=650{ }^{\circ} \mathrm{C}$ ). For the specific geometry of the Pt-1 electrodes, this value implies a penetration depth for the surface influence zone that is of the order of $0.6 \mu \mathrm{m}$ (Fig. 5a), and a fractional reaction contribution to the interfacial resistance, $f_{\mathrm{rxn}}$, that is close to one for all oxygen partial pressures and temperatures examined. Thus, the electrode resistance is dominated under all relevant conditions by the

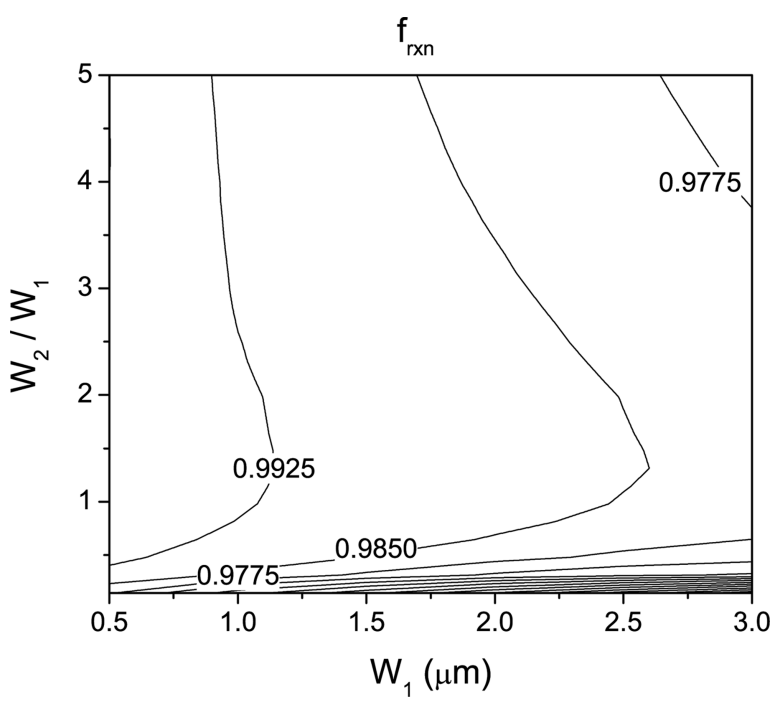

Fig. 10 Quasi-sensitivity-analysis of the fractional surface resistance, obtained by fitting the model parameters to the Pt-1 experimental data while fixing the current collector dimensions to various values. Maximum and minimum $f_{\mathrm{rxn}}$ in this plot are 1.00 and 0.88 , respectively. $T=650^{\circ} \mathrm{C}$ and $p_{\mathrm{O}_{2}}=4.1 \times 10^{-26} \mathrm{~atm}$. reaction occurring on the ceria surface, whereas the sizable penetration depth for the cross-plane electronic current suggests that any surface features are unlikely to directly influence the electron diffusion-drift behavior.

Approximating a grid-like porous metal on ceria as line patterns could lead to errors in the fraction of MIEC|gas interface and the in-plane diffusion length. We performed a quasi-sensitivity-analysis on $f_{\mathrm{rxn}}$ with respect to the geometric parameters (Fig. 10), by fitting $k^{0}$ to the experimental data (of Pt-1) at various fixed values of $W_{1}$ and $W_{2}$. Under the wide range of geometries analyzed, we find that $f_{\text {rxn }}$ is never lower than 0.88. Given that the fractional surface resistance is far larger than 0.5 (the case where surface reaction and electron diffusion-drift are equally co-limiting), we conclude that errors in determining the current collector geometry will not shift the electrode reactions from one that is limited by surface reaction to one that is limited by in-plane diffusion.

\section{Conclusions}

The two-dimensional electrochemical model developed here to describe transport in a mixed ionic and electronic conductor (MIEC) with patterned metal current collectors reveals several important features of the electrochemical behavior. The mixed-conducting nature of the cell gives rise to two types of electron fluxes: (1) cross-plane electronic current that flows between the metal current collectors on either side of the cell, and (2) in-plane electronic current that flows between the reaction site on the MIEC|gas interface and the metal. The macroscopic interfacial resistance, corresponding to what would be measured experimentally, can be readily decomposed into two terms: the diffusion-drift resistance associated with the in-plane electronic current and the electrochemical reaction resistance associated with the charge transfer reaction on the MIEC surface.

In the limit of infinitely fast surface kinetics, the expected interfacial impedance on SDC15 (which is set solely by the electron diffusion-drift behavior) for metal current collectors with micron-scale features is $\sim 10^{-2} \Omega \mathrm{cm}^{2}$ at $650{ }^{\circ} \mathrm{C}$ and $1 \times 10^{-25}$ atm oxygen partial pressure. Because the measured interfacial impedance is about two-orders of magnitude higher, electron diffusion-drift is ruled out as the rate-determining step for hydrogen electro-oxidation on this MIEC. The experimental observation of an interfacial impedance that nevertheless scales with $p_{\mathrm{O}_{2}}^{1 / 4}$ has motivated the use of a global reaction rate expressed as $k=k^{0} p_{\mathrm{O}_{2}}^{-1 / 4}$. Experimental data for three distinct measurements using two different types of electrodes imply $k^{0}\left(T=650{ }^{\circ} \mathrm{C}\right)$ of approximately $6 \times 10^{-7} \Omega^{-1} \mathrm{~cm}^{-2}$ atm $^{\frac{1}{4}}$ and yield consistent interfacial impedance values after appropriate normalization for surface features. While the detailed mechanistic steps for the surface reaction remain unknown, the observed oxygen partial pressure dependence of $k$ suggests that the electron concentration (which scales $p_{\mathrm{O}_{2}}^{-1 / 4}$ ) may play a role. The computational methods employed here are both rapid and accurate because they take advantage of modern, adaptive-mesh techniques. Because of the subtle interaction between the cross- and 
in-plane electronic currents and the varying penetration depth of the in-lane current, it would not be possible to explore the features of interest here using simplified, one-dimensional models.

\section{List of symbols}

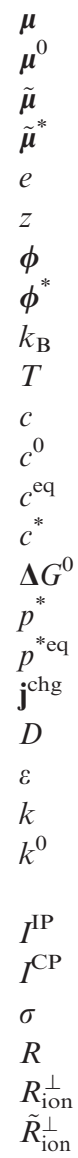

$\boldsymbol{\mu} \quad$ chemical potential

$\boldsymbol{\mu}^{0} \quad$ chemical potential at standard state

$\tilde{\boldsymbol{\mu}}$ electrochemical potential

$\tilde{\boldsymbol{\mu}}^{*} \quad$ reduced electrochemical potential

$e \quad$ elementary charge

$z \quad$ formal charge

$\phi \quad$ electric potential

$\Delta G^{0}$

$\tilde{R}_{\text {ion }}^{* \perp}$

$R_{\text {rxn }}$

$R_{\text {eon-DD }}$

$f_{\text {surf }}$

$W_{1}$

$W_{2}$

l

$d_{\mathrm{SIZ}}$

eq

(1)

IP

CP

ion

eon

dop

red

non-dimensional electric potential

Boltzmann constant

temperature

carrier concentration

carrier concentration at standard state

equilibrium carrier concentration

non-dimensional carrier concentration

standard Gibbs free energy of reaction

gas activity

equilibrium gas activity

charge flux

diffusion coefficient

permittivity

surface reaction rate-constant

partial-pressure independent surface reaction

rate-constant

in-plane current

cross-plane current

conductivity

resistance

electrode resistance

electrode resistance normalized by the total

electrode area $\left(W_{1}+W_{2}\right)$; identical to the

conventional, macroscopically defined

interfacial resistance

electrode resistance normalized by the active

reaction area $\left(W_{2}\right)$

surface reaction resistance (tildes indicate

normalization-see $\tilde{R}_{\text {ion }}^{\perp} \& \tilde{R}_{\text {ion }}^{* \perp}$ )

in-plane electron diffusion-drift resistance

fractional surface reaction resistance

half of metal current collector width

half of MIEC width exposed to gas

half of the sample thickness

depth of the surface-influence-zone

equilibrium

dimensionless

perturbed

in-plane

cross-plane

ionic species (oxygen vacancy)

electronic species (polaron)

dopant species

reduction (specifically for standard Gibbs free $g$

rxn

eon-DD gas (specifically for standard Gibbs free energy for gas phase reaction of oxygen, hydrogen and water) surface reaction (MIEC|gas interface)

electron diffusion-drift (between MIEC|metal and MIEC $\mid$ gas interface)

energy of oxide reduction)

\section{Appendix 1}

We utilize a linear boundary condition to describe ion transfer across the MIEC|gas interface:

$$
\left.\mathbf{j}_{\text {ion }}^{\text {chg }} \cdot \hat{\mathbf{y}}\right|_{\Gamma_{5}}=-k\left(\left.\tilde{\mu}_{\text {ion }}^{*(1)}\right|_{\Gamma_{5+}}-\left.\tilde{\mu}_{\text {ion }}^{*(1)}\right|_{\Gamma_{5}}\right)
$$

where $k$ is the surface reaction rate-constant (normalized by the MIEC|gas interfacial area), $\Gamma_{5+}$ denotes the exposed surface of the MIEC, $\Gamma_{5}$ denotes the MIEC immediately beneath the surface, and $\hat{\mathbf{y}}$ is a unit vector in the direction perpendicular to the interface. The electrochemical potential of the ionic species on the exposed surface $\Gamma_{5}+$ can be evaluated as follows. By the requirement of local equilibrium, the following is true everywhere in the MIEC and on the $\Gamma_{5}$ surface:

$$
\frac{1}{4 e} \mu_{\mathrm{O}_{2}}+\tilde{\mu}_{\mathrm{ion}}^{*}-\tilde{\mu}_{\mathrm{eon}}^{*}=0
$$

where $\mu_{\mathrm{O}_{2}}$ is the chemical potential of gaseous oxygen and it is assumed there is no gas-phase concentration gradient near the MIEC|gas interface. For a small perturbation this is readily achieved by maintaining a sufficiently high gas flow rate. Under a small bias eqn (47), specifically on the exposed surface of the oxide, becomes:

$$
\left.\tilde{\mu}_{\text {ion }}^{*(1)}\right|_{\Gamma_{5+}}-\left.\tilde{\mu}_{\text {eon }}^{*(1)}\right|_{\Gamma_{5+}}=0
$$

The assumption of reversibility of the electron transfer reaction implies

$$
\left.\tilde{\mu}_{\mathrm{eon}}^{*(1)}\right|_{\Gamma_{5+}}-\left.\tilde{\mu}_{\mathrm{eon}}^{*(1)}\right|_{\Gamma_{5}}=0
$$

Substituting eqn (48) and (49) into eqn (46), the chemical reaction rate can be rewritten as:

$$
\left.\mathbf{j}_{\text {ion }}^{\text {chg }} \cdot \hat{\mathbf{y}}\right|_{\Gamma_{5}}=-k\left(\left.\tilde{\mu}_{\text {eon }}^{*(1)}\right|_{\Gamma_{5}}-\left.\tilde{\mu}_{\text {ion }}^{*(1)}\right|_{\Gamma_{5}}\right)
$$

Substituting the expression for $\tilde{\mu}_{i}^{*(1)}$ of eqn (20) into eqn (50), the ion flux across the interface is given as:

$$
\left.\mathbf{j}_{\text {ion }}^{\text {chg }} \cdot \hat{\mathbf{y}}\right|_{\Gamma_{5}}=\left.\frac{k_{\mathrm{B}} T}{e} k\left(c_{\text {eon }}^{*(1)}+\frac{c_{\text {ion }}^{*(1)}}{2}\right)\right|_{\Gamma_{5}}
$$

This expression can be further reduced by inserting the electroneutrality approximation:

$$
\left.\mathrm{j}_{\text {ion }}^{\text {chg }} \cdot \hat{\mathbf{y}}\right|_{\Gamma_{5}}=\left.\frac{k_{\mathrm{B}} T}{e} k c_{\text {eon }}^{*(1)}\left(1+\frac{c_{\text {eon }}^{\text {eq }}}{4 c_{\text {ion }}^{\text {eq }}}\right)\right|_{\Gamma_{5}}
$$

To complete the boundary condition expression, it is recognized that the current is conserved across the MIEC|gas interface. That is, the flux of species $i$ leaving the MIEC|gas 
interface as a result of diffusion-drift as given by eqn (10), must equal the flux of that species injected as a result of the electrochemical reaction at the interface:

$$
-\left.\frac{\left(e z_{i}\right)^{2} D_{i} c_{i}}{k_{\mathrm{B}} T} \frac{\partial \tilde{\mu}_{i}^{*(1)}}{\partial y}\right|_{\Gamma_{5}}=\left.\mathbf{j}_{i}^{\mathrm{chg}} \cdot \hat{\mathbf{y}}\right|_{\Gamma_{5}}
$$

Furthermore, electroneutrality implies that the ionic current generated by the chemical reaction must be balanced by the electronic current:

$$
\left.\mathrm{j}_{\text {ion }}^{\text {chg }} \cdot \hat{\mathbf{y}}\right|_{\Gamma_{5}}=-\left.\mathbf{j}_{\text {eon }}^{\text {chg }} \cdot \hat{\mathbf{y}}\right|_{\Gamma_{5}}
$$

Substituting eqn (20) and (54) into eqn (53) and writing out the equation for both ionic and electronic carriers gives:

$$
\begin{gathered}
\left.D_{\text {eon }} c_{\text {eon }}^{\text {eq }}\left(\frac{\partial c_{\text {eon }}^{*(1)}}{\partial y}-\frac{\partial \phi^{*(1)}}{\partial y}\right)\right|_{\Gamma_{5}}=-\left.\frac{k_{\mathrm{B}} T}{e^{2}} k c_{\text {eon }}^{*(1)}\left(1+\frac{c_{\text {eon }}^{\mathrm{eq}}}{4 c_{\text {ion }}^{\mathrm{eq}}}\right)\right|_{\Gamma_{5}} \\
\left.D_{\text {ion }} c_{\text {ion }}^{\text {eq }}\left(\frac{c_{\text {eon }}^{\mathrm{eq}}}{c_{\text {ion }}^{\mathrm{eq}}} \frac{\partial c_{\text {eon }}^{*(1)}}{\partial y}+4 \frac{\partial \phi^{*(1)}}{\partial y}\right)\right|_{\Gamma_{5}}=-\left.\frac{k_{\mathrm{B}} T}{e^{2}} k c_{\text {eon }}^{*(1)}\left(1+\frac{c_{\text {eon }}^{\mathrm{eq}}}{4 c_{\text {ion }}^{\mathrm{eq}}}\right)\right|_{\Gamma_{5}}
\end{gathered}
$$

Combining eqn (55) and (56) and rearranging gives the following boundary conditions which describe the first-order chemical reaction taking place on the MIEC|gas interface:

$$
\begin{aligned}
& \left.\frac{\partial c_{\text {eon }}^{*(1)}}{\partial y}\right|_{\Gamma_{5}}=-\left.\frac{k_{\mathrm{B}} T}{e^{2}} \frac{k c_{\text {eon }}^{*(1)}}{4 D_{\text {ion }} c_{\text {ion }}^{\text {eq }}}\left(1+\frac{4 D_{\text {ion }} c_{\text {ion }}^{\text {eq }}}{D_{\text {eon }} c_{\text {eon }}}\right)\right|_{\Gamma_{5}} \\
& \left.\frac{\partial \phi^{*(1)}}{\partial y}\right|_{\Gamma_{5}}=-\left.\frac{k_{\mathrm{B}} T}{e^{2}} \frac{k c_{\text {eon }}^{*(1)}}{4 D_{\text {ion }} c_{\text {ion }}^{\text {eq }}}\left(1-\frac{D_{\text {ion }}}{D_{\text {eon }}}\right)\right|_{\Gamma_{5}}
\end{aligned}
$$

\section{Appendix 2}

The partial differential equations and the boundary conditions solved numerically are listed below:

$$
\begin{aligned}
\nabla^{2} c_{\mathrm{eon}}^{*(1)} & =0 \\
\nabla^{2} \phi^{*(1)} & =0 \\
\left.\phi^{(1)}\right|_{\Gamma_{1}} & =0 \\
\left.c_{\mathrm{eon}}^{*(1)}\right|_{\Gamma_{1}} & =0 \\
\left.\frac{\partial c_{\mathrm{eon}}^{*(1)}}{\partial x}\right|_{\Gamma_{2}, \Gamma_{3}} & =0
\end{aligned}
$$

$$
\begin{aligned}
& \left.\frac{\partial \phi^{*(1)}}{\partial x}\right|_{\Gamma_{2}, \Gamma_{3}}=0 \\
& \left.\frac{\partial c_{\text {eon }}^{*(1)}}{\partial y}\right|_{\Gamma_{4}}=-\left.4 \frac{c_{\text {ion }}^{\text {eq }}}{c_{\text {eon }}^{\text {eq }}} \frac{\partial \phi^{*(1)}}{\partial y}\right|_{\Gamma_{4}} \\
& \left.\phi^{*(1)}\right|_{\Gamma_{4}}=1 \mathrm{~V}\left(\frac{k_{\mathrm{B}} T}{e}\right)^{-1} \\
& \left.\frac{\partial c_{\text {eon }}^{*(1)}}{\partial y}\right|_{\Gamma_{5}}=-\left.\frac{k_{\mathrm{B}} T}{e^{2}} \frac{k c_{\text {eon }}^{*(1)}}{4 D_{\text {ion }} c_{\text {ion }}^{\text {eq }}}\left(1+\frac{4 D_{\text {ion }} c_{\text {ion }}^{\text {eq }}}{D_{\text {eon }} c_{\text {eon }}}\right)\right|_{\Gamma_{5}} \\
& \left.\frac{\partial \phi^{*(1)}}{\partial y}\right|_{\Gamma_{5}}=-\left.\frac{k_{\mathrm{B}} T}{e^{2}} \frac{k c_{\text {eon }}^{*(1)}}{4 D_{\text {ion }} c_{\text {ion }}^{\text {eq }}}\left(1-\frac{D_{\text {ion }}}{D_{\text {eon }}}\right)\right|_{\Gamma_{5}}
\end{aligned}
$$

The boundary condition embodied in eqn (66) requires some comment. In an experimental system, ultimately one fixes the electrochemical potential of electrons and not the electric field at the MIEC/metal interface. In the present analysis, however, the electric field rather than the electron electrochemical potential is taken to be the fixed quantity. In a onedimensional linear system, the computational results for these two choices are identical in terms of extracted quantities such as impedance. In the present two-dimensional, linear system, there is a small numerical difference between the two. In the case in which the electrochemical potential is fixed, then the electronic current will only flow in a direction normal to the MIEC/metal interface. In the case in which the electric potential is fixed, then the electronic current need not be orthogonal to the interface. The computed differences between these two situations are sufficiently small that there is little impact of the choice on the global conclusions. In the absence of detailed knowledge of the physical properties of the metals, a fixed electric potential along the MIEC|metal interface is assumed largely to avoid the counter-intuitive restriction on electronic current flow implied by a fixed electron electrochemical potential. Moreover, a fixed electric potential is more readily compared to a one-dimensional solution in that the electric field in the metal becomes one-dimensional.

\section{Appendix 3}

Strictly, the in-plane electron diffusion-drift resistance is given by

$$
R_{\text {eon-DD }}=\frac{\left\langle\tilde{\mu}_{\mathrm{eon}}^{*}\right\rangle_{\varphi\left(\Gamma_{4}\right)}-\left\langle\tilde{\mu}_{\mathrm{eon}}^{*}\right\rangle_{\Gamma_{5}}}{I_{\mathrm{eon}}^{\mathrm{IP}}}
$$

where the numerator is the difference between the reduced electrochemical potential for oxygen vacancies averaged (as indicated by the brackets) over the MIEC|gas interface and the potential for electrons averaged over portions of the MIEC $\mid$ metal interface (indicated by $\varphi\left(\Gamma_{4}\right)$ ) that is accessed by the in-plane diffusion-drift current. As illustrated in Fig. 3(a), although electrons will originate from the entire MIEC|gas interface, they will reach only a portion of the MIEC|metal interface, due to interactions with the cross-plane electronic 
current coming from the current collector on the opposite side of the cell. To properly compute $\varphi\left(\Gamma_{4}\right)$, one should follow the trajectories of the electrons migrating in the in-plane direction. However, error in $R_{\text {eon-DD }}$ by making the approximation $\varphi\left(\Gamma_{4}\right) \approx \Gamma_{4}$ is less than $11 \%$ under the simulation conditions of this work. For computational simplicity, we average the electrochemical potentials of electrons over the entire MIEC|metal interface when calculating the electrode resistance.

\section{Acknowledgements}

This work was supported by the Stanford Global Climate Energy Project and the Office of Naval Research under grant N00014-05-1-0712. Additional support was provided by the Gordon and Betty Moore Foundation through the Caltech Center for Sustainable Energy Research and by the National Science Foundation through the Caltech Center for the Science and Engineering of Materials, a Materials Research Science and Engineering Center (DMR-052056). The authors are grateful to Evan Brown for performing image analysis.

\section{References}

1 S. B. Adler, Chem. Rev., 2004, 104, 4791-4844.

2 S. P. Jiang and S. H. Chan, J. Mater. Sci., 2004, 39, 4405-4439.

3 A. Trovarelli, Catal. Rev. Sci. Eng., 1996, 38, 439-520.

4 A. J. Jacobson, Chem. Mater., 2010, 22, 660-674.

5 Y.-H. Huang, R. I. Dass, Z.-L. Xing and J. B. Goodenough, Science, 2006, 312, 254-257.

6 Z. P. Shao and S. M. Haile, Nature, 2004, 431, 170-173.

7 S. M. Haile, Acta Mater., 2003, 51, 5981-6000.

8 S. B. Adler, J. A. Lane and B. C. H. Steele, J. Electrochem. Soc., 1996, 143, 3554-3564.

9 F. S. Baumann, J. Fleig, H.-U. Habermeier and J. Maier, Solid State Ionics, 2006, 177, 1071-1081.

10 F. Ciucci, PhD Thesis, California Institute of Technology, 2009.

11 F. Ciucci, W. C. Chueh, S. M. Haile and D. G. Goodwin, arXiv:0707.0835 [Chemical Physics], 2009.

12 F. Ciucci, Y. Hao and D. G. Goodwin, Phys. Chem. Chem. Phys., 2009, 11, 11243-11257.
13 G. W. Coffey, L. R. Pederson and P. C. Rieke, J. Electrochem. Soc., 2003, 150, A1139-A1151.

14 R. Das, D. Mebane, E. Koep and M. Liu, Solid State Ionics, 2007, 178, 249-252.

15 H. M. Deng, M. Y. Zhou and B. Abeles, Solid State Ionics, 1994, 74, 75-84.

16 J. Descure, Y. Bultel, L. Dessemomd and E. Siebert, Solid State Ionics, 2005, 176, 235-244.

17 J. Fehribach and R. OHayre, SIAM J. Appl. Math., 2009, 70, 510-530.

18 J. Fleig, J. Power Sources, 2002, 105, 228-238.

19 J. Fleig and J. Maier, J. Eur. Ceram. Soc., 2004, 24, 1343-1347.

20 R. D. Green, C.-C. Liu and S. B. Adler, Solid State Ionics, 2008, 179, 647-660.

21 J. Jamnik and J. Maier, J. Electrochem. Soc., 1999, 146, 4183-4188.

22 J. Jamnik and J. Maier, Phys. Chem. Chem. Phys., 2001, 3, $1668-1678$.

23 W. Lai and S. M. Haile, J. Am. Ceram. Soc., 2005, 88, 2979-2997.

24 M. L. Liu, J. Electrochem. Soc., 1998, 145, 142-154.

25 Y. Lu, C. Kreller and S. B. Adler, J. Electrochem. Soc., 2009, 156, B513-B525.

26 D. S. Mebane and M. Liu, J. Solid State Electrochem., 2006, 10, 575-580.

27 D. S. Mebane, Y. Liu and M. Liu, J. Electrochem. Soc., 2007, 154, A421-A426.

28 A. M. Svensson, S. Sunde and K. Nisancioglu, J. Electrochem. Soc., 1998, 145, 1390-1400.

29 F. S. Baumann, J. Maier and J. Fleig, Solid State Ionics, 2008, 179, $1198-1204$.

30 W. C. Chueh, W. Lai and S. M. Haile, Solid State Ionics, 2008, 179, 1036-1041.

31 E. P. Murray, T. Tsai and S. A. Barnett, Nature, 1999, 400, 649-651.

32 S. Park, J. M. Vohs and R. J. Gorte, Nature, 2000, 404, 265-267.

33 T. Kobayashi, S. Wang, M. Dokiya, H. Tagawa and T. Hashimoto, Solid State Ionics, 1999, 126, 349-357.

34 R. J. Panlener, R. N. Blumenthal and J. E. Garnier, J. Phys. Chem. Solids, 1975, 36, 1213-1222.

35 E. Ruiz-Trejo and J. Maier, J. Electrochem. Soc., 2007, 154, 583-587.

36 H. L. Tuller and A. S. Nowick, J. Phys. Chem. Solids, 1977, 38, 859-867.

37 S. Wang, H. Inaba and H. Tagawa, J. Electrochem. Soc., 1997, 144, 4076-4080.

38 H. C. Chang and G. Jaffe, J. Chem. Phys., 1952, 20, 1071-1077.

39 A. Quarteroni and A. Valli, Numerical Approximation of Partial Differential Equations, Springer-Verlag, New York, 1994.

$40 \mathrm{~F}$. Hecht and O. Pironneau, FreeFem ++ , Universite Pierre et Marie Curie, 2007.

41 T. A. Davis, Assoc. Comput. Mach., Trans. Math. Software, 2004, 30, 196-199. 